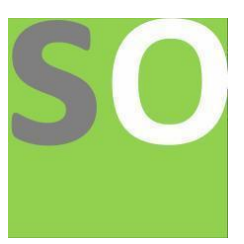

Article title: ADMINISTRATION SYSTEM FOR END TO END LUXURY APARTMENT MANAGEMENT SOFTWARE Authors: Kevin Joseph[1]

Affiliations: cse, karunya institute of technology and science[1]

Orcid ids: 0000-0002-1655-760X[1]

Contact e-mail: kevinjoseph1501@gmail.com

License information: This work has been published open access under Creative Commons Attribution License http://creativecommons.org/licenses/by/4.0/, which permits unrestricted use, distribution, and reproduction in any medium, provided the original work is properly cited. Conditions, terms of use and publishing policy can be found at https://www.scienceopen.com/.

Preprint statement: This article is a preprint and has not been peer-reviewed, under consideration and submitted to ScienceOpen Preprints for open peer review.

Links to data: worksbot

DOI: 10.14293/S2199-1006.1.SOR-.PPROHQ1.v1

Preprint first posted online: 23 April 2021 


\section{ADMINISTRATION SYSTEM FOR END TO END \\ LUXURY APARTMENT MANAGEMENT SOFTWARE}

a project report submitted by

KEVIN JOSEPH (REG. NO: UR17CS130)

in partial fulfillment for the award of the degree of

BACHELOR OF TECHNOLOGY

in

COMPUTER SCIENCE AND ENGINEERING

under the supervision of

DR. M. ROSHNI THANKA

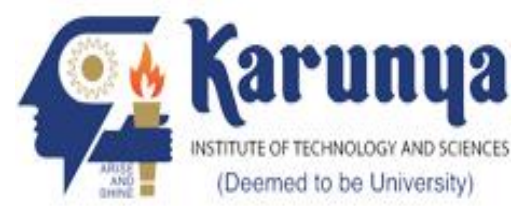

DEPARTMENT OF COMPUTER SCIENCE AND ENGINEERING SCHOOL OF ENGINEERING AND TECHNOLOGY

KARUNYA INSTITUTE OF TECHNOLOGY AND SCIENCES (Declared as Deemed-to-be-University under Sec-3 of the UGC Act, 1956)

Karunya Nagar, Coimbatore - 641 114, India.

APRIL 2021 


\section{BONAFIDE CERTIFICATE}

Certified that this project report "ADMINISTRATION SYSTEM FOR END TO END LUXURY APARTMENT MANAGEMENT SOFTWARE" is the bonafide work of "KEVIN JOSEPH (REG. NO: UR17CS130)" who carried out the project work under my supervision.

SIGNATURE

\section{Dr. J. Immanuel Johnraja}

Head of the Department

Department of Computer Science and Engineering
SIGNATURE

\section{Dr. M Roshni Thanka}

\section{Supervisor}

Assistant Professor

Department of Computer Science and

Engineering

Submitted for the Project Viva Voce held on.

Examiner 


\section{ACKNOWLEDGEMENT}

First and foremost, I praise and thank ALMIGHTY GOD for giving me the will power and confidence to carry out my project.

I am grateful to my beloved founders Late Dr. D.G.S. Dhinakaran, C.A.I.I.B, Ph.D., and Dr. Paul Dhinakaran, M.B.A., Ph.D., for their love and always remembering me in their prayers.

I extend my thanks to Dr. P. Mannar Jawahar, Ph.D., honorable vice chancellor, Dr. E. J. James, Ph.D., Dr. Ridling Margaret Waller, Ph.D., and Dr. T. Lazar Mathew, Ph.D., honorable Pro-Vice Chancellor(s) and Dr. R. Elijah Blessing, Ph.D., respected registrar for giving me the opportunity to carry out this project.

I am thankful to Dr. G. Prince Arulraj, M.E., Ph.D., Dean (Engineering \& Technology) for his support and encouragement.

I would like to place our heart-felt thanks and gratitude to Dr. J. Immanuel Johnraja, Ph.D., HOD, Department of Computer Science and Engineering for his encouragement and guidance.

I am grateful to my guide, Dr. M Roshni Thanka, Assistant Professor, Department of Computer Science and Engineering for her valuable support, advice and encouragement.

I also thank all the staff members of the Department for extending their helping hands to make this project work a success.

I would also like to thank my parents and all my friends who have prayed and helped me during the project work. 


\section{ABSTRACT}

A majority of unpleasant issues faced by landlords and tenants seem to grow due to lack of communication. Late rent payments turns into an eviction. A non-functional $\mathrm{AC}$ or a broken window becomes a reason to break a lease. Fortunately, building a positive relationship doesn't take much effort and it all starts with one basic idea: communication.

This project is entitled as "Administration System for end to end luxury apartment management software", which is a software for managing highly customizable multiple apartments, flat, building or any kind of real estate properties.

The software is to ease property management burdens on small and independent landlords. The challenge was to conceptualize and develop a simple, streamlined management tool for smaller property owners. The tool needed to be able to manage marketing for the property, encourage on-time payments by tenants, keep track of transactions, and handle maintenance requests.

The key technologies used for creating the software are HTML, CSS, Bootstrap, JavaScript, Python, MySQL and jQuery.

In Existing security system there is authentication at user level the user id and password submitted by user is verified at login process end if the user id and password exist then the user would be able to access the system. But some smart user usually uses SQL injection in order to violate the security of database using wild character of SQL. They sometime use SQL statement too in order to get the

login process confused. They pass the sub query of sql in password field instead of password in order to get the confirmation. Here we have to develop a secure system for authentication access and apply SQL INJECTION attack to check its security. 


\section{CONTENTS}

S.no

1

1.1

1.2

1.3

1.4

2

2.1

2.2

2.3

2.4

2.4 .1

2.4 .2

2.4 .3

2.4 .4

Chapter 1: Introduction

Page no.

Topic

1

Objective

1-2

Company Profile

2

Problem Statement

Scope of project

Chapter 2: System Analysis

Existing System

Proposed System

5

Usecase analysis

6

Requirement Specification

8

Functional requirements

Non-functional requirements

9-10

Hardware requirements

10

Software requirements

10

3

Chapter 3: System Design

Detailed design

$10-17$

3.2

Design of methodology

17-20

3.3

Modules

3.4

Database design

22

3.4.1

Database entities

22

4

Chapter 4: System implementation

Module implementation

24

4.2

Test Driven Development

29

5

Chapter 5: Conclusion and future scope 


\section{LIST OF FIGURES}

\section{S.no Figure}

no.

$\begin{array}{ll}1 & 2.3\end{array}$

$1 \quad 3.1 .1$

$3 \quad 3.1 .2$

$4 \quad 3.1 .3$

$5 \quad 3.2$
Topic

Use Case Diagram

Architecture Diagram

Data Flow Diagram

Sequence Diagram

Agile Model
Page no.

7

11

$12-14$

$15-16$

17 


\section{LIST OF TABLES}

\section{S.no Figure}

no.

1

$2 \quad 1.6 .4$

$3 \quad 3.4 .2 .1$

$4 \quad 3.4 .2 .2$

$5 \quad 3.4 .2 .3$

$6 \quad 3.4 .2 .4$
Topic

Software Requirements

Hardware Requirements

Unit Information

Floor Information

Tenant Information

Employee Information

\section{Page no.}

9

9

22

22

23

23 


\section{LIST OF SYMBOLS AND ABBREVIATIONS}

SQL

HTML

CSS

BFM

GFM

SDLC

HTTP
Structured Query Language

HyperText Markup Language

Cascading Style Sheets

Behavioral Feature Matrix

Grammatical Feature Matrix

Software Development Life Cycle

Hypertext Transfer Protocol 


\section{CHAPTER 1}

\section{INTRODUCTION}

In this era of modern technology renting an apartment is within our hands. But the challenges after that is time consuming and misleading. With the current change in outlook in innovative field, there is an earnest need to embrace and appreciate the force of innovation.

Property management remains watchful to confront the difficulties of progress by utilizing another methodology that encourages simple administration of properties. Subsequently there is need to develop a management system for the property and this board framework that can improve on work for the property owners and tenants with the goal that all their work can be productive and successful.

The challenge was to conceptualize and develop a simple, streamlined management tool for property owners. The tool needed to be able to manage marketing for the property, encourage ontime payments by tenants, keep track of transactions, and handle maintenance requests. The system not only supports the owners but even ensures the tenants are having a pleasant environment to stay.

\subsection{PROJECT OBJECTIVE}

The goal of the project was to ultimately offer three separate personalized dashboards - one for admin, one for owner and one for tenants. The tool needed to eliminate owner's need to keep paper records, track down late payments, or personally deal with maintenance requests. Once functional, owner would never again need to keep paper records on tenants, leases, payments, invoices, or maintenance requests. The software would also remind tenants of due dates and automatically tack on fees for late payment.

For tenants, the tool would be where they signed leases, referred back to signed documents, and renew a lease when applicable. They will be able to complaint about anything that is improper in their property. 
They can easily contact the owner or the employee present in the property who is in charge of the maintenance without waiting for a call back. The details of the tenants staying in a particular apartment owner can be displayed and also the details of the management committee responsible for the tenants.

In addition to managing day-to-day contact between landlords and tenants, the software also needed to be able to generate periodic and on-demand reports, searchable property, tenant name, date range, invoice type, and payment type.

\subsection{COMPANY PROFILE}

Worksbot is a best solutions and development company specializing in Artificial Intelligence (AI) \& Machine Learning technologies. Worksbot was created to leverage technology to empower creative minds all over the world.

Worksbot has delivered more than 600 projects, to over 200 clients worldwide. Many Fortune 100 companies in retail, banking, Automobile, healthcare and so on are powered by our solutions to streamline and leverage their growth.

\subsection{PROBLEM STATEMENT}

Property management system for this organization uses traditional method of keeping records of the client's files. This manual record keeping in the organization has been characterized by a lot of problems, such as:

a. Lack of skill in interpretation of reports from the activities of the organization.

b. Data losses: loss of data perhaps would happen if all information only kept inside paper on.

c. Data redundancies: abundant and repetition data also perhaps will happen.

d. No database to store information: by using manual system, loss of data perhaps will happen. 
e. No backup and security: still information to contemporary system perhaps have been trespassed easily or stolen, this is because of the insecurity in the manual system used in the organization.

\subsection{SCOPE OF PROJECT}

- This software is a web-based application that allows landlords to communicate electronically with tenants. The software contains all records with ease and also save the data of the owner and the tenant.

- The main application of the software is that it can be used by apartment owners and landlords at property sites in an efficient manner. This will save a lot of time and power and thus will benefit the user.

- The application is a modern, scalable service for independent landlords and tenants that simplifies the process, ultimately saving both landlords and tenant's time, money, and effort. As a computer based system it will be easier to search, obtain and delete data from the backend.

\section{- Integrated \& dynamic prediction of SQL injection using machine learning}

Machine Learning combines computer science, mathematics, and statistics. Statistics is essential for drawing inferences from the data. Mathematics is useful for developing machine learning models and finally, computer science is used for implementing algorithms. However, simply building models is not enough. You must also optimize and tune the model appropriately so that it provides you with accurate results. Optimization techniques involve tuning the hyper parameters to reach an optimum result.

Current day web services provide a lot more services than simple content delivery. Web based model of computing has been subject several attacks such as cross-site scripting \& SQL injection. SQL Injection Attacks are comparatively recent threat to privacy, integrity \& accessibility of all online requests $\&$ their technical infrastructure, secretarial for practically fourth of internet vulnerabilities. 
This proposed system has represented types of attacks \& classification of SQL injection attack. Next survey based on research done represented in tabular form. After that discussed about pattern locked proposed model \& conclusion then future scope, suggested way for researchers for preventing SQL injection attacks. 


\section{CHAPTER 2 \\ SYSTEM ANALYSIS}

Collecting and interpreting facts, identifying the problems, and decomposition of a system into its components. System analysis is conducted for the purpose of studying a system or its parts in order to identify its objectives. It is a problem solving technique that improves the system and ensures that all the components of the system work efficiently to accomplish their purpose.

\subsection{EXISTING SYSTEM}

The existing system has many loopholes and limitations. It has too much manual work from filling a form to preparing a document, this increases burden on the workers which doesn't yield the result it should. If any modification has to be made it is completely manual and is error prone.

The present systems does not use advanced technologies with minimum user interaction which causes more hustle and less satisfaction for the users. It contains less functionalities for the tenants. A proper verification is not maintained in these systems.

\section{Limitations of existing system are:}

- Unprotected guest data.

- Takes time in retrieving single data from the database.

- It only provides text based interface which is not as user friendly as Graphical user interface.

\subsection{PROPOSED SYSTEM}

The proposed software is a web application that gives all the features provided by the existing systems, with enhanced specifications. This software provides equal importance to the needs of the owner and the tenant. It will provide smooth functioning for all the modules and the data is stored in a sorted manner. 
With the use of scripting and programming language an interesting software has been made for the user. Various pages are created about the apartment, owner, tenant, employee, maintenances, rent payment details, complaints and also the visitors to the apartments.

The input of proposed system is a large number of Web access logs. In order to train our detection model, we first preprocess the logs, e.g., filtering and decoding. Then we get the programming patterns covered in dataset. After customizing the log fields, we then design and implement a grammar recognizer and behavior miner to extract grammatical and behavioral features in log files, respectively. Finally, based on the generated two kinds of training sets and testing sets: grammatical feature matrix (GFM) and behavioral feature matrix (BFM).

URL encoding is used to ensure that the server can successfully receive data input from users with different IP addresses, while URL decoding is used to restore the encoded result to the original form of the information, which is provided by the user on the local browser. In our work, we decode the logs according to the encoding mechanism, e.g., the space is encoded as "\%20", nontext characters are represented by "\%XY", and characters are represented by 6-digit hexadecimal characters and separated by $\% " .$.

\section{Advantageous of proposed system}

- Owner and Tenant details are secured with login details.

- The system being an online software, will give accurate information regarding the property which helps to view all the information directly from anywhere.

- Faster data accessing from the database.

\subsection{USECASE ANALYSIS}

A use case analysis is the essential structure for social occasion use necessities for new software or undertaking to be finished. The essential objectives of a use case analysis are: planning a framework from the client's point of view, conveying framework conduct in the client's terms, and indicating all remotely noticeable practices. 
In the use case diagram shown in Figure 2.3, the use cases address the functionalities of the property owner and tenant. Aside from registration, login and documentation which is normal among owner and tenant, landowner will refresh lease, utilities, crisis data which is introduced on the inhabitant's UI. Tenant will have functionalities, for example, detailing issue's and payment details which is shown on owner's screen.

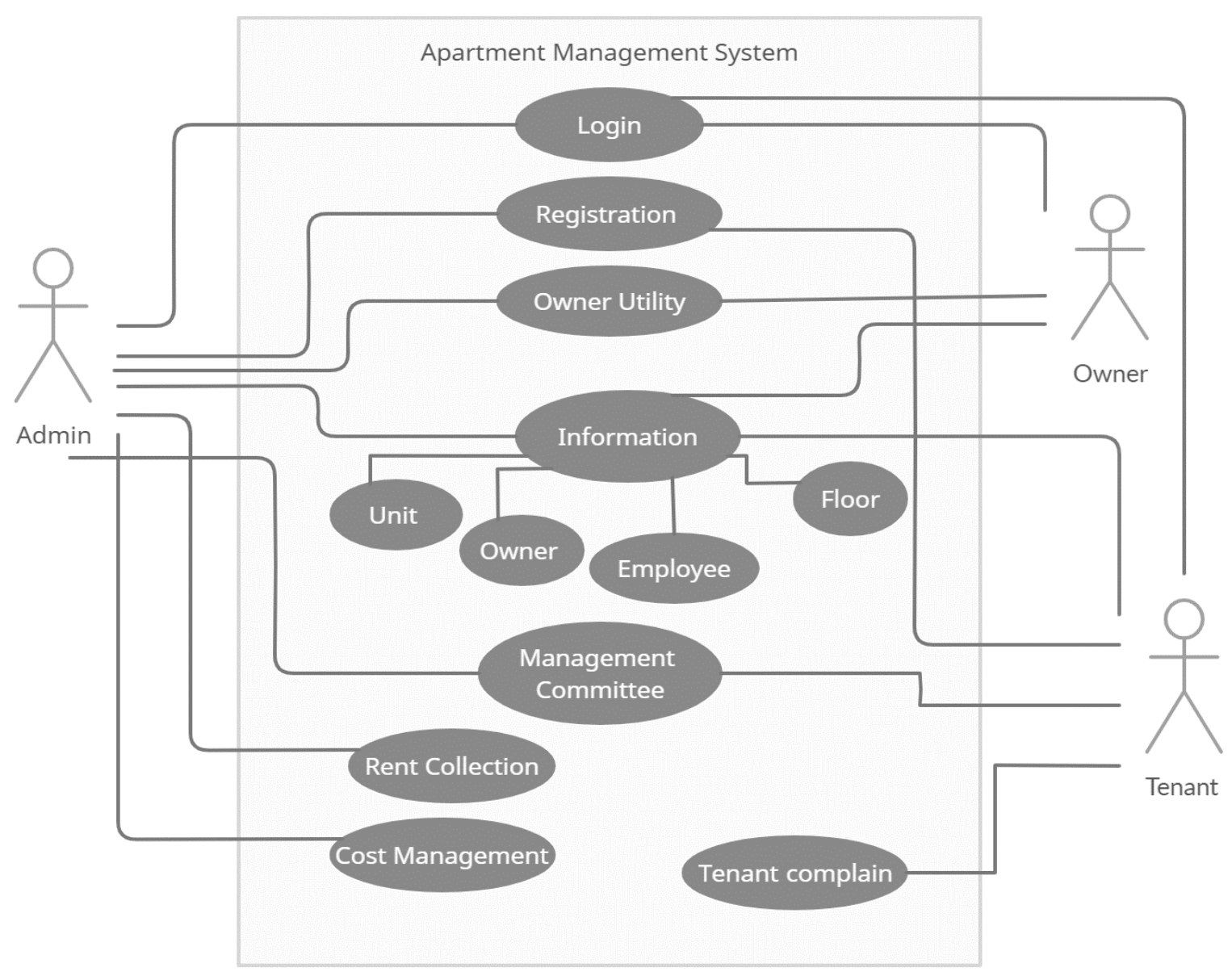

Fig 2.3 Use Case Diagram

Actors: Admin, Owner, Tenant

Use cases: Login, Registration, Owner Utility, Informations,

Management committee, Rent collection, Cost management, Tenant complain

Actors in the diagram are Admin, Owner and Tenant. The admin control every activity in the system. He access the database for any information and to complete the needs of the tenant and also to keep the owner updated. 


\subsection{REQUIREMENT SPECIFICATION}

Clear requirements help development teams create the right product. And a software requirements specification helps to lay the groundwork for product development. It describes what the software will do and how it will be expected to perform. It also describes the functionality the product needs to fulfill all stakeholders (business, users) needs.

\subsubsection{FUNCTIONAL REQUIREMENTS}

- Secure login to the system using the web application for admin, owner and user.

- Allow administrator to add and delete houses, tenant details.

- Allow the administrator to search and edit data in the database.

- The admin provides and controls the floor, unit and owner information.

- The details of the employee working and the tenant staying can be edited by the admin.

- The authenticity of the rent paid can be verified by the admin for the owner.

- Maintenance details and complaints can be displayed.

- Management committee, i.e. for each unit is displayed for the tenants.

- Visitors to each apartment can be added.

\subsubsection{NON-FUNCTIONAL REQUIREMENTS}

- Improve Search option:

Search option should be rich enough to provide the property details to user. Search option should search in the whole database.

Efficient search option attracts the user to interact more with the system.

- Support Multi-User:

Nowadays, every system work in an environment of multi-users. As per requirement of the system, the system is developed in multi user's environment.

- Fast:

Search option should be fast enough to produce a result of the search in seconds. Every module should to compatible with other modules. There should not be any lag or delay in processing the data. 
- Available (24X7):

Property Management System should be available to clients $24 X 7$. The user can access the system whenever they need to access, wherever they want to access provided having an internet connection and device to access.

- The system improves on the efficiency of information, storage and retrieval.

- It is easy to learn and use.

- It is flexible, safe and convenient.

\subsubsection{HARDWARE REQUIREMENTS}

3 Table 1.6.3 Hardware Requirements

\begin{tabular}{||l||l||}
\hline Processor & $\begin{array}{l}\text { Intel(R) Core(TM) i7-7500U CPU @ 2.70GHz 2.90 } \\
\text { GHz }\end{array}$ \\
\hline Ram & $8.00 \mathrm{~GB}(7.89 \mathrm{~GB}$ usable) \\
\hline Hard disk drive & 1024 GB \\
\hline System type & 64-bit operating system, x64-based processor \\
\hline $\begin{array}{l}\text { Windows } \\
\text { Specification }\end{array}$ & $\begin{array}{l}\text { Edition - Windows 10 Pro } \\
\text { Version - 1909 }\end{array}$ \\
\hline
\end{tabular}

\subsubsection{SOFTWARE REQUIREMENTS}

Table 1.6.4 Software Requirements

\begin{tabular}{||l||l||}
\hline Front-End & HTML5, CSS3, Bootstrap, JavaScript \\
\hline Back-End & Java (Spring/Spring Boot) or Python (Django/Flask) \\
\hline Database & MySQL \\
\hline Client server tool & jQuery \\
\hline
\end{tabular}




\section{CHAPTER 3}

\section{SYSTEM DESIGN}

The database was updated each time the administrator; add, deletes or deletes data on the system. It's only the administrator who has access to the system to view or make changes when necessary. The system was designed to allow the administrator to view, edit, delete and add data to the database.

\subsection{DETAILED DESIGN}

\subsubsection{ARCHITECTURE}

An architectural diagram is a diagram of a system that is used to abstract the overall outline of the software system and the relationships, constraints, and boundaries between components. It is an important tool as it provides an overall view of the physical deployment of the software system and its evolution roadmap.

An architectural diagram must serve several different functions. To allow relevant users to understand a system architecture and follow it in their decision-making, we need to communicate information about the architecture. Architectural diagrams provide a great way to do this. To put down some major functions, an architectural diagram needs to:

- Break down communication barriers

- Reach a consensus

- Decrease ambiguity 


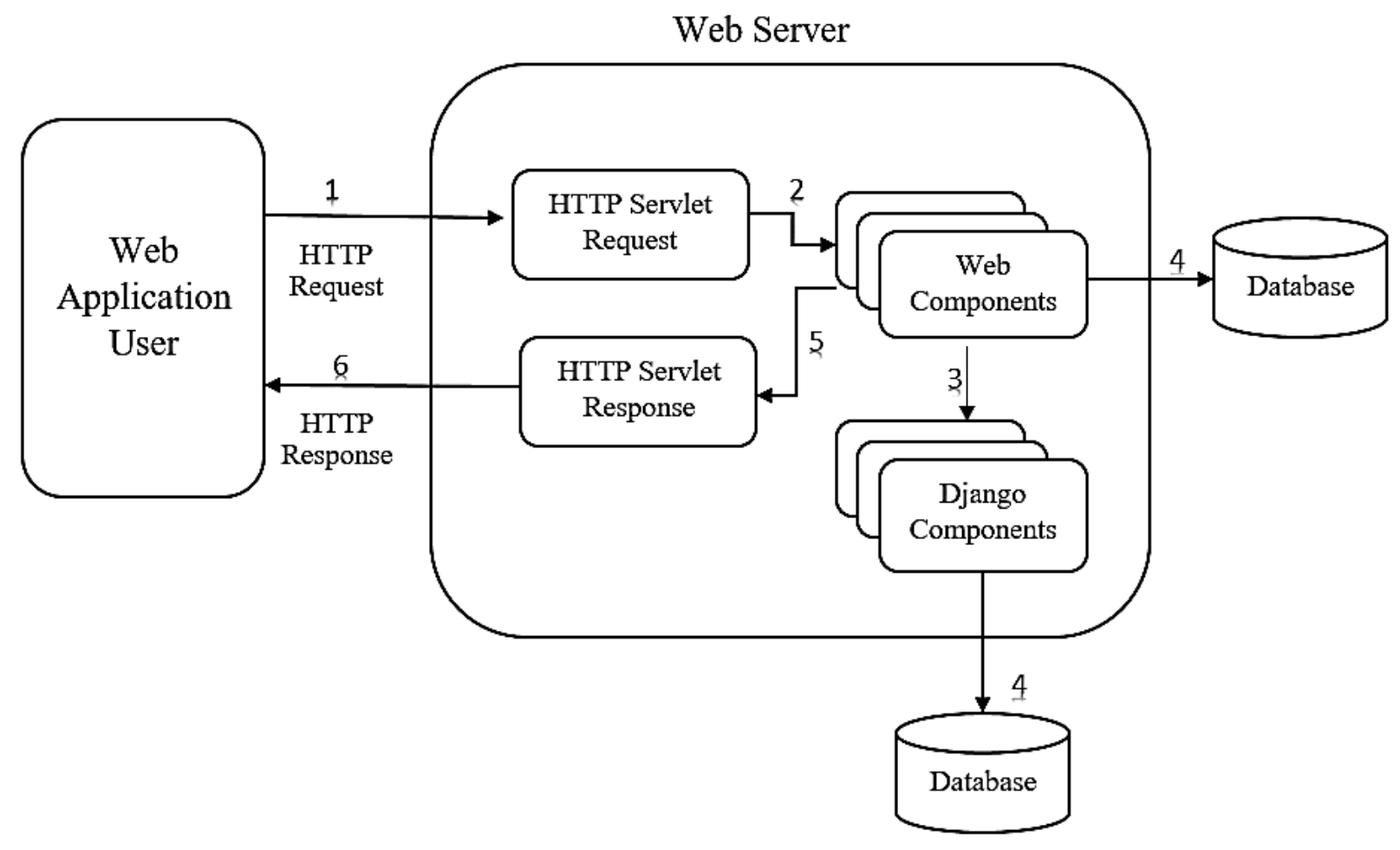

Fig 3.1.1 Architecture Diagram

- A HTTP request is a text string generated by the client and sent to the server containing the specifications of the resource the client is asking for. The resource the client wants to interact with is communicated through the URL that is sent with the request.

- An HTTP response is what is sent by a server to a client in response to an HTTP request.

- HTTP responses also include a message body. If the client is a browser, the HTML body is generally an HTML page that the browser can then render.

- Once the client receives the HTTP response, the browser will render the HTTP response body message. This message is either the requested resource (for example, a web page) or display a message relating to status code if the request wasn't successful. 


\subsubsection{DATA FLOW DIAGRAM}

A data flow diagram (DFD) illustrates how data is processed by a system in terms of inputs and outputs. Its focus is on the flow of information, where data comes from, where it goes and how it gets stored.

The system supports multiple access of users at the same time and hence the data flow is shown from both sides.

Level 0:
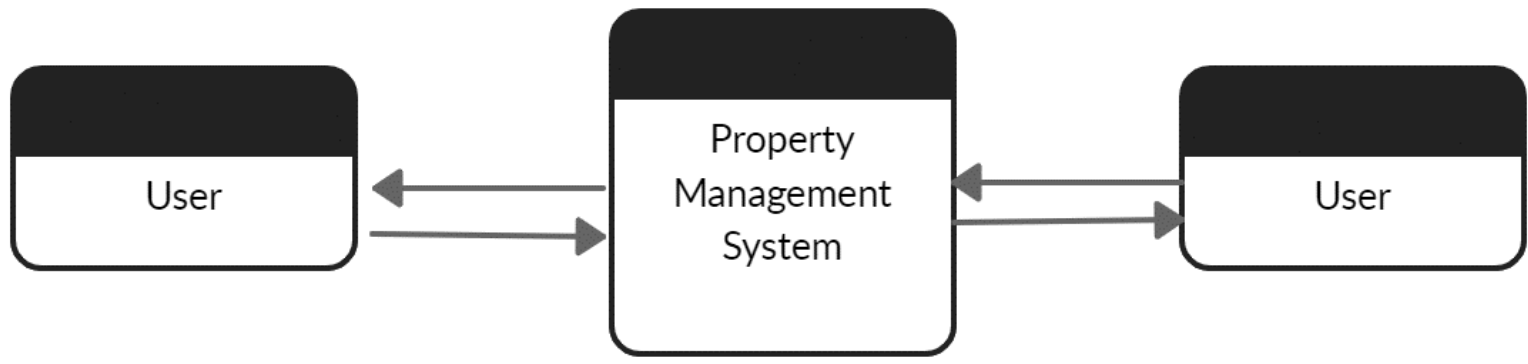

. Level 0 indicates the multiple users accessing the software from different areas at different time. The user can either be an admin, Owner or a tenant.

\section{Level 1:}

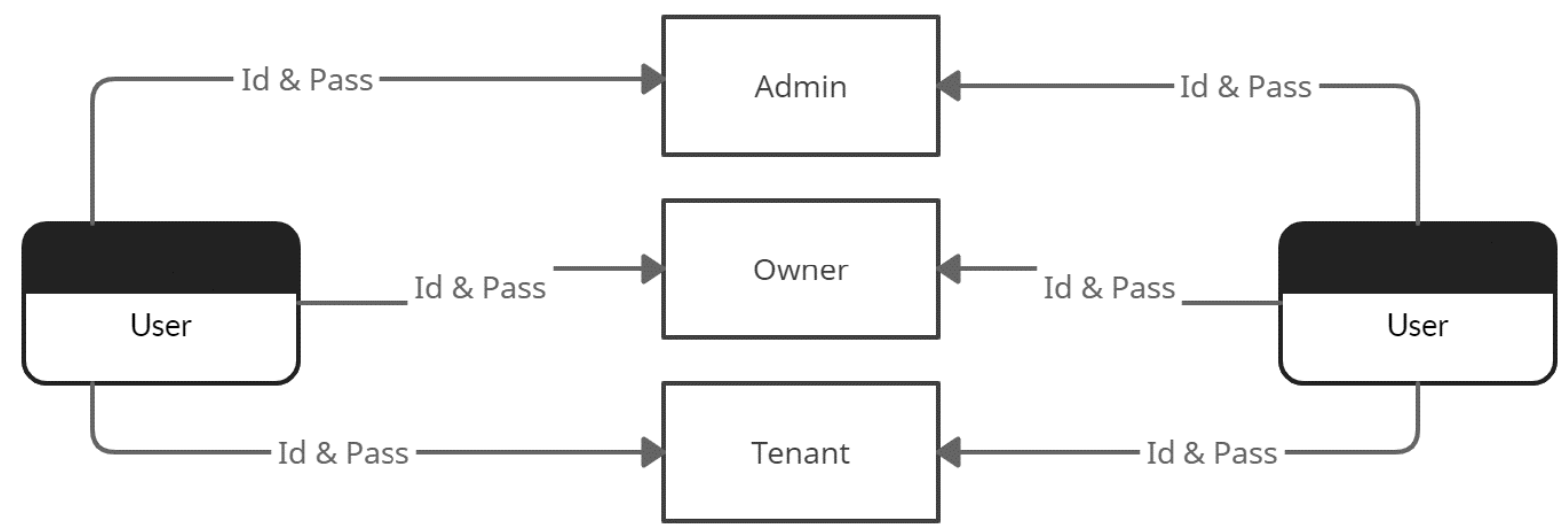

For enhanced security the user must login using their ID and password to access any pages in the software. 


\section{Level 2:}

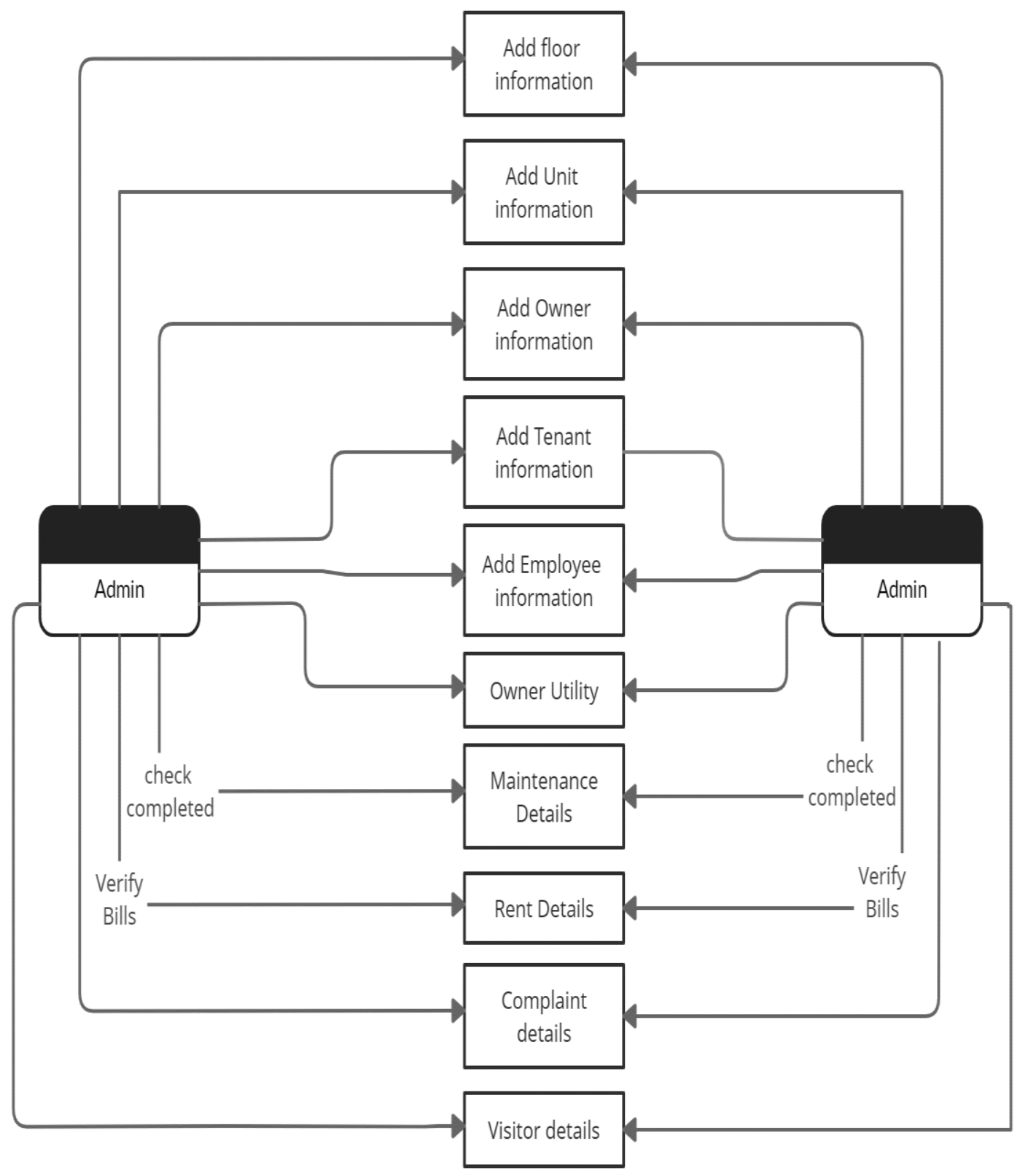

The admin has direct access to all the pages in the web application, he can manage any database of the owner or the tenant.

He can verify any payment or maintenance details from his side, supporting the owner and tenant at the same time. 


\section{Level 2:}

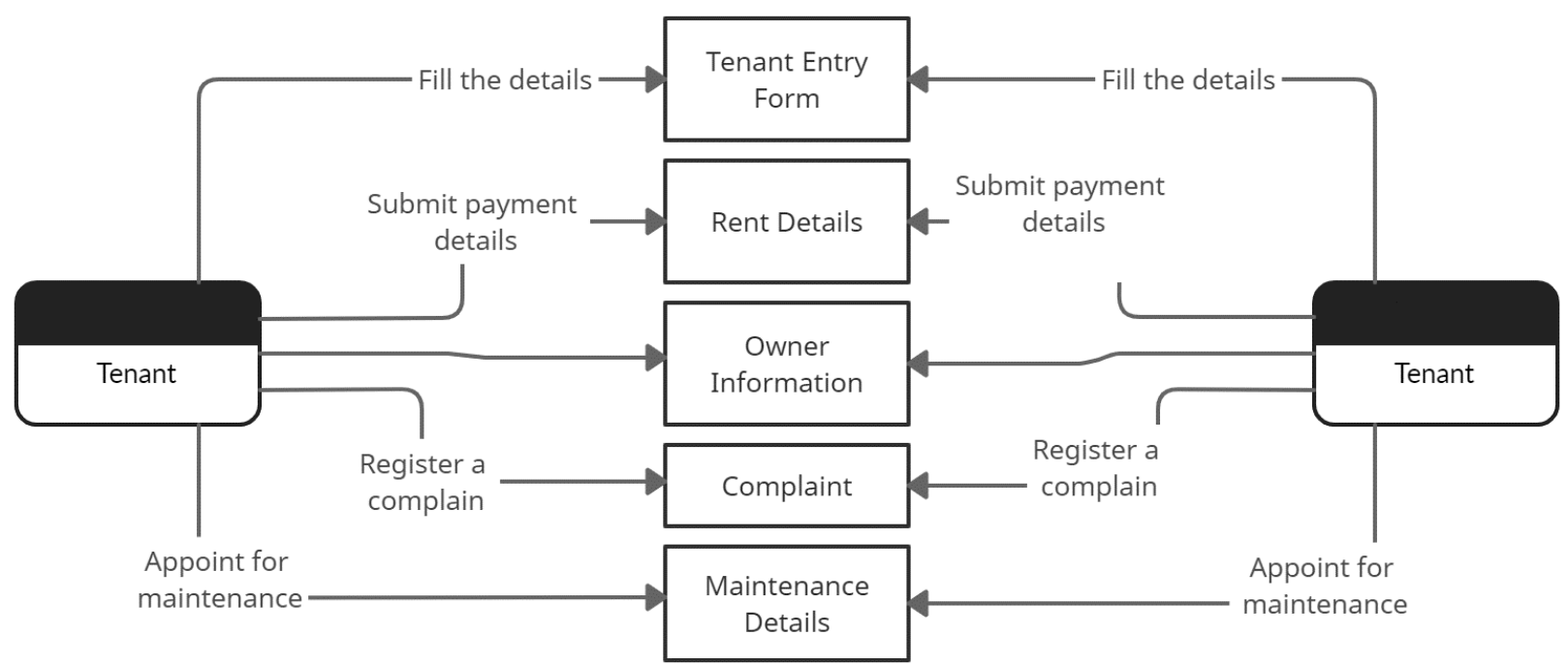

\section{Level 2:}

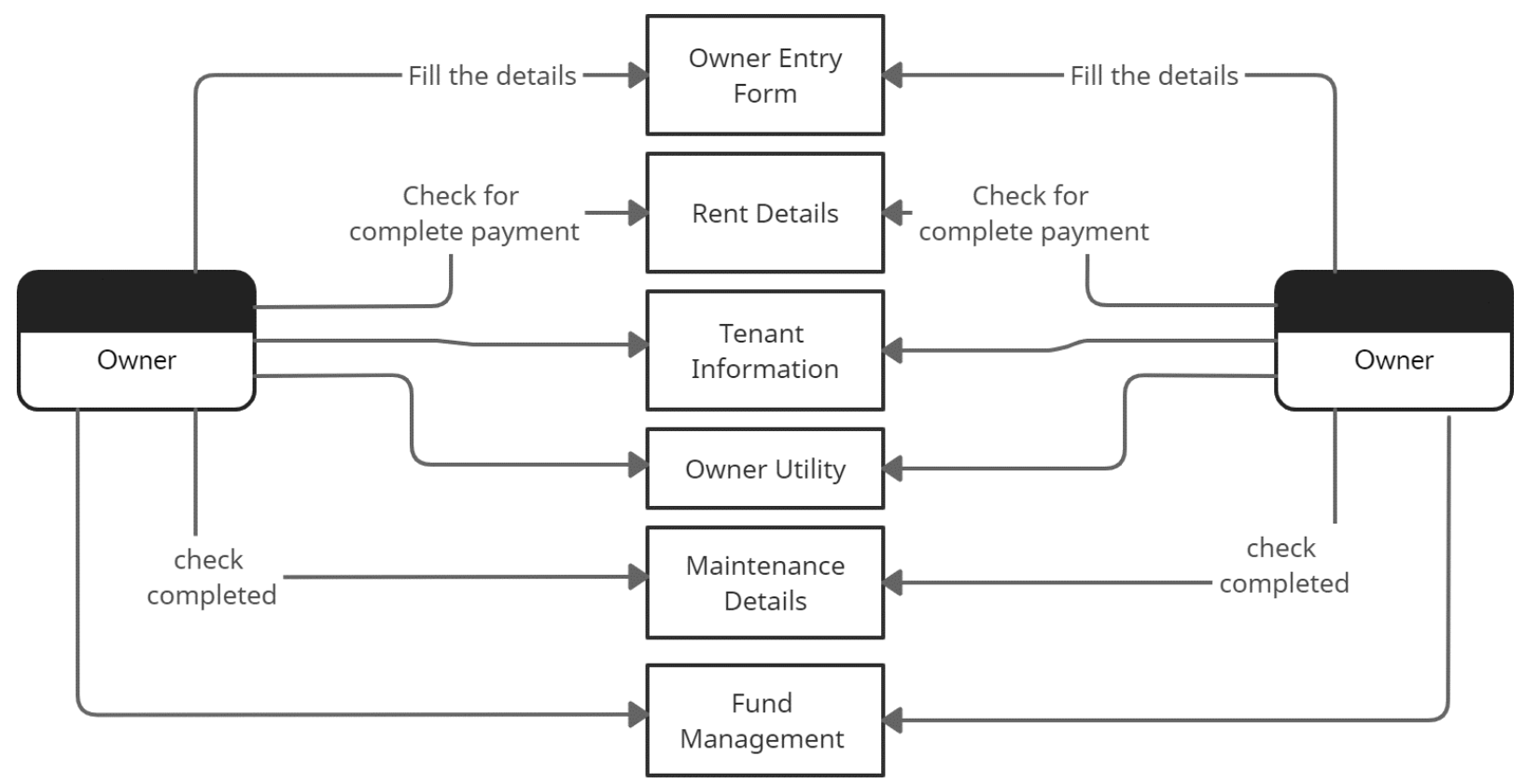

Fig 3.1.2 Data flow diagram 


\subsubsection{SEQUENCE DIAGRAM}

Sequence Diagrams are interaction diagrams that detail how operations are carried out. They capture the interaction between objects in the context of a collaboration. Sequence Diagrams are time focus and they show the order of the interaction visually by using the vertical axis of the diagram to represent time what messages are sent and when.

Sequence Diagrams captures:

- The interaction that takes place in a collaboration that either realizes a use case or an operation.

- High-level interactions between user of the system and the system, between the system and other systems, or between subsystems.

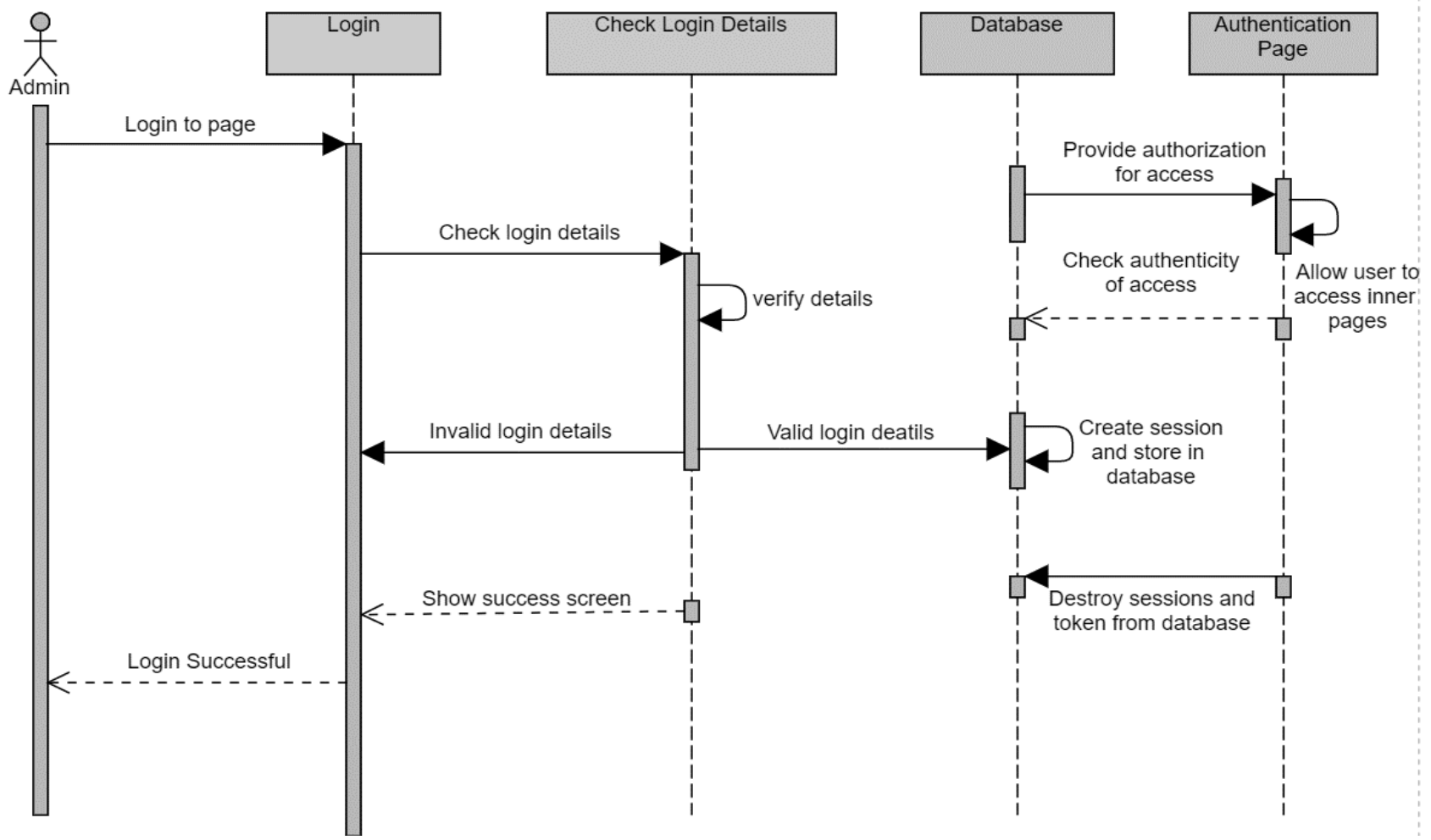




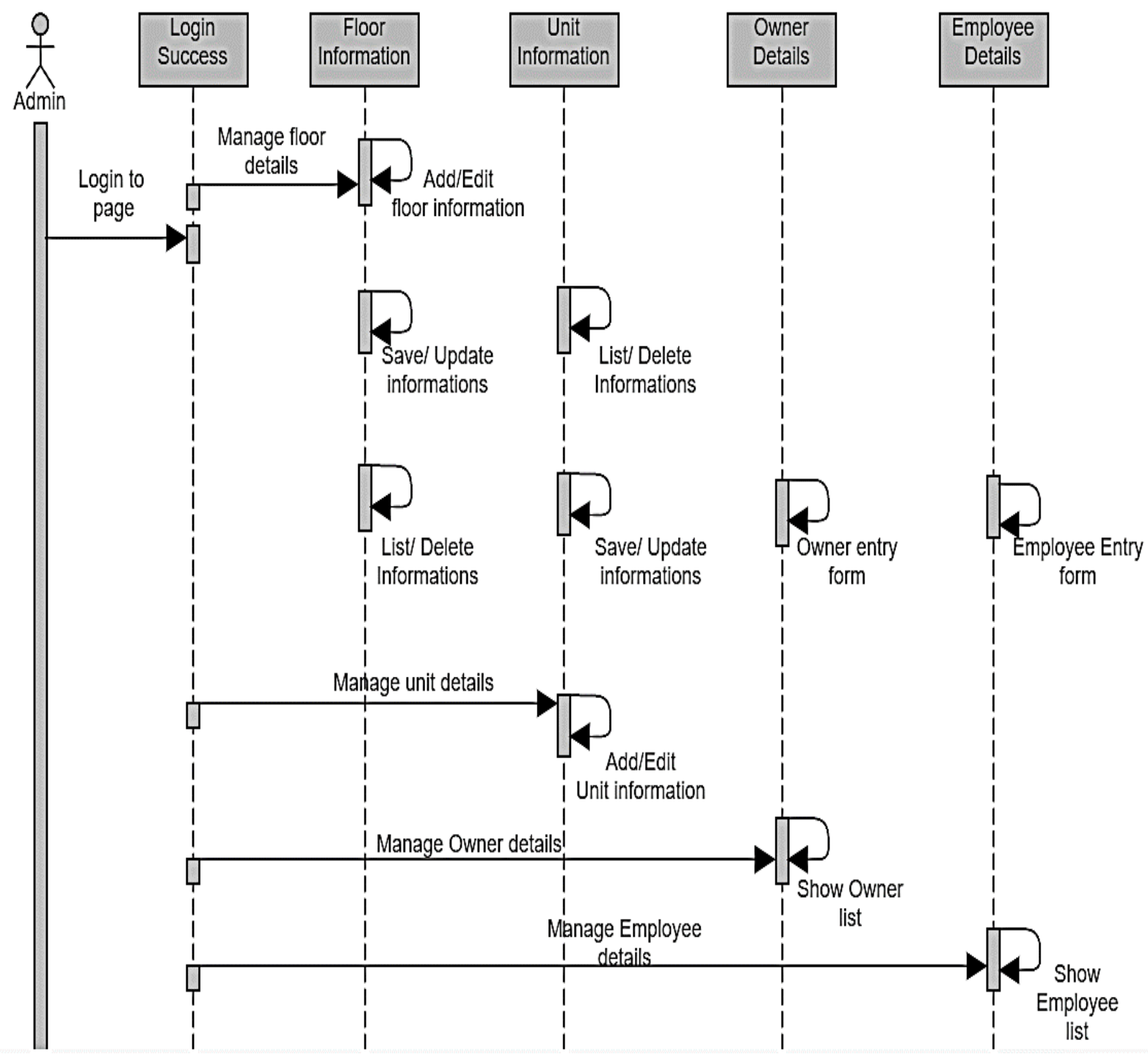

Fig 3.1.3 Sequence Diagram 


\subsection{DESIGN OF METHODOLOGY}

The systems development life-cycle concept applies to a range of hardware and software configurations, as a system can be composed of hardware only, software only, or a combination of both. If the SDLC concept is adhere to, the programmer will derive good software that is error free that will satisfied all the needs in a good condition. The phases are as follows:

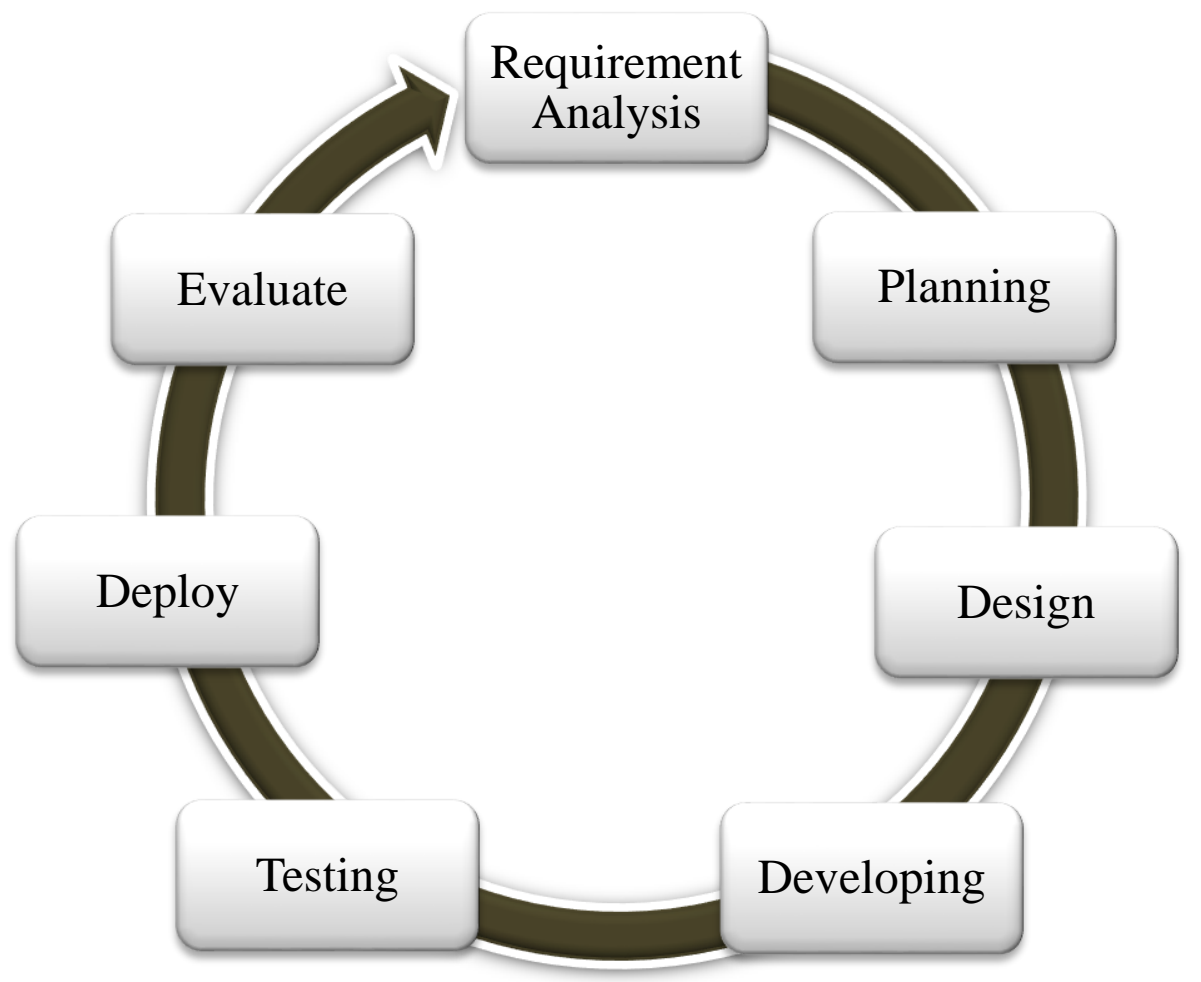

Fig 3.2 Agile Model

The Agile software development methodology is one of the easiest and compelling cycles to transform a dream for a business need into programming arrangements. Agile is a term used to portray software development moves toward that utilize constant arranging, learning, improvement, group coordinated effort, transformative turn of events, and early conveyance. It urges adaptable reactions to change.

The agile software development emphasizes on four core values.

1. Individual and team interactions over processes and tools

2. Working software over comprehensive documentation

3. Customer collaboration over contract negotiation 
4. Responding to change over following a plan

The sequential phases described in the agile model are:

1. Requirement Analysis:

All possible requirements are captured in product requirement documents.

Carry out a study to gain an understanding of the owners and tenant's current method of property management and their problems experienced in this method through interviews and observations.

Gathered requirement such as software like the programming language to use, database model and hardware needed such as laptop, printers etc.

2. Planning:

In the planning phase the requirements gathered in the requirements phase, are used to create report definitions and layouts, screen definitions and layouts, data element definitions, workflow diagrams, and security matrices.

3. Design:

In the design phase the logical model developed in the analysis phase, is used to develop a physical model of the application. The stage will have an overall design of the system architecture and physical design which includes User Interface and Database design.

Identifying any faults before moving onto the next stage is very important. The design specification that is used in the next stage of implementation is according to the output from the design stage.

\section{Developing:}

The development phase is about writing code and converting design documentation into the actual software within the software development process. This stage of SDLC is generally the longest as it's the backbone of the whole process.

In this stages the coding is done as per the design specification and physical model. Once all the objects are created for the system, they are gathered together to create a working application. 
5. Testing:

This encompasses three testing stages; component testing, requirements testing, and acceptance testing.

In all testing stages, defects are identified and returned to the development/coding phase for correction. Here it is verified that the application is error free and all the bugs are fixed before moving to the next stage.

6. Deploy:

The application is deployed on the servers and given to the clients - either for the demo or the real use. Further cycles update the generally introduced programming, presenting new highlights and settling bugs.

After completing all the test procedures, the product is executed for the client purposes and verified.

7. Evaluate:

Once all previous development phases are complete, the Product Owner gathers the Development Team once again and reviews the progress made towards completing the requirements.

The deployed application is maintained by scheduled verifications and backups. Any modification that is requested by the user or due to any bug that was not found initially is corrected here for better performance of the system.

It is then presented to the organization and the development cycle begins again.

\section{Benefits of using agile methodology:}

Stakeholder Engagement:

Agile provides multiple opportunities for stakeholder and team engagement - before, during, and after each Sprint.

\section{Transparency:}

An Agile approach provides a unique opportunity for clients to be involved throughout the project, from prioritizing features to iteration planning and review sessions to frequent software builds containing new features. 
Early and Predictable Delivery:

By using time-boxed, fixed schedule Sprints of 1-4 weeks, new features are delivered quickly and frequently, with a high level of predictability.

Predictable Costs and Schedule:

Because each Sprint is a fixed duration, the cost is predictable and limited to the amount of work that can be performed by the team in the fixed-schedule time box.

Allows for Change:

While the team needs to stay focused on delivering an agreed-to subset of the product's features during each iteration, there is an opportunity to constantly refine and reprioritize the overall product backlog.

Focuses on Business Value:

By allowing the client to determine the priority of features, the team understands what's most important to the client's business, and can deliver the features that provide the most business value.

Focuses on Users:

Agile commonly uses user stories with business-focused acceptance criteria to define product features

Improves Quality:

By breaking down the project into manageable units, the project team can focus on highquality development, testing, and collaboration.

\subsection{MODULES}

- Property Administration

- Property Master (Owner)

- Residence and Leasing

- Accounting and Reporting

- SQL Injection Detection 
Property administrators:

Property administrators system perform administrative duties and co-ordinate activities related to the management and rental of investment property and real estate on behalf of property and strata property owners.

\section{Property Master (Owner):}

Property Master Role is meant for the actual owners of the apartment who can oversee the tenants and maintenance of their beloved place.

\section{Residence and Leasing:}

The Residential and Leasing maintains the accessibilities that are available in the Apartments, manages the benefits that are there in the Apartment. Checks the vacancy of the rooms and provides a friendly and time saving experience for the tenants.

Accounting and Reporting:

The aim is to provide an accounting Features for Improved Business Insight and DecisionMaking. It also helps in Managing Rentable Items with perfect and timely maintenances.

\section{SQL Injection Detection:}

The Proposed system uses both signature-based and anomaly-based detection models to identify threats and attacks on the system. Signatures are carefully selected to implement the signaturebased model. Anomaly-based detection is based on the number of times a user attempted to access the system, regardless of whether any SQL-I patterns have been detected. Proposed system is case-insensitive while trying to use the signature-based detection method to detect SQL-I attacks. The proposed system deals with White Space Manipulation attack by removing any white space before comparing text with known SQL-I attack patterns.

The proposed system deals with Comments attack by looking for comment characters in the submitted text. The String Concatenation attack is dealt with by looking for the concatenation operation characters or CONCAT function. The keyword "UNION" is searched for by the proposed system in case an attack tries to perform the UNION Injection attack. The system will also look for binary, hexadecimal, and decimal characters in the submitted text to catch 
instances of this SQL-I attack variation. Sample patterns may be found in the SQLI_PATTERNS table described in the proposed system database schema.

Even when no SQL-I attack pattern is detected in the submitted form text, the proposed system monitors the frequency of the login attempts to implement the anomaly-based detection method. When the number of visits has exceeded a predetermined threshold, the system automatically blocks the visitor for a time. It is significant to note that the screen the user sees when he or she has entered an incorrect password or when an SQL- I pattern is detected in the text matches.

\subsection{DATABASE DESIGN}

Database design defines the database structure used for planning, storing, and managing information. Accuracy in data can only be accomplished if a database is designed to store only valuable and necessary information.

A well-designed database is imperative in guaranteeing information consistency, eliminating redundant data, efficiently executing queries, and improving the performance of the database. Meticulously designing a database saves you from wasting time and getting frustrated during the database development phase. A good database design also allows you to easily access and retrieve data whenever needed.

\subsubsection{DATABASE ENTITIES}

Table 3.4.2.1 Unit Information

\section{Unit Information}

\section{Entity Name}

Floor number

\section{Data type}

int

Table 3.4.2.2 Floor Information

\section{Floor Information}

\begin{tabular}{|l|l|}
\hline Entity Name & Data type \\
\hline Floor number & int \\
\hline Unit number & int \\
\hline
\end{tabular}


Table 3.4.2.3 Tenant Information

\begin{tabular}{|l|l|}
\hline \multicolumn{2}{|c|}{ Tenant Information } \\
\hline Entity Name & Data type \\
\hline Tenant name & Varchar \\
\hline Email & varchar \\
\hline Password & varchar \\
\hline Contact & int \\
\hline Address & varchar \\
\hline NID & int \\
\hline Issue date & varchar \\
\hline Rented unit number & int \\
\hline Advance payment & int \\
\hline Payment per month & Int \\
\hline Status & Varchar \\
\hline
\end{tabular}

Table 3.4.2.4 Employee Information

\begin{tabular}{|l|l|}
\hline \multicolumn{2}{|c|}{ Employee Information } \\
\hline Entity Name & Data type \\
\hline Employee name & Varchar \\
\hline Email & varchar \\
\hline Contact & varchar \\
\hline Address & varchar \\
\hline NID & int \\
\hline
\end{tabular}




\section{CHAPTER 4}

\section{SYSTEM IMPLEMENTATION}

\subsection{MODULE IMPLEMENTATION}

\section{Technologies used:}

The back end includes Python (Django) and database management system as MYSQL while in the front end the web programming was done with HTML5 (hypertext makeup language), Java script, CSS3 (cascading Style Sheet), Pandas and bootstrap.

HTML:

Hypertext Markup Language (HTML) is the standard markup language for documents designed to be displayed in a web browser.

CSS:

Cascading Style Sheets (CSS) is a style sheet language used for describing the presentation of a document written in a markup language such as HTML.CSS is designed to enable the separation of presentation and content, including layout, colors, and fonts.

JavaScript:

JavaScript is a scripting language. It is different from Java language. It is object-based, lightweight, cross-platform translated language. It is widely used or client-side validation. The javaScript Translator (embedded in the browser) is responsible for translating the JavaScript code for the web browser

Bootstrap:

Bootstrap is a free and open-source CSS framework directed at responsive, mobile-first frontend web development. It contains CSS- and JavaScript-based design templates for typography, forms, buttons, navigation, and other interface components. 
SQLite:

SQLite is a database engine. It is software that allows users to interact with a relational database. In SQLite, a database is stored in a single file - a trait that distinguishes it from other database engines. This fact allows for a great deal of accessibility: copying a database is no more complicated than copying the file that stores the data, sharing a database can mean sending an email attachment.

Python:

Python was created by Guido van Rossum, and released in 1991. It is a general-purpose computer programming language. It is a high-level, object-oriented language which can run equally on different platforms such as Windows, Linux, UNIX, and Macintosh. It is widely used in data science, machine learning and artificial intelligence domain.

Pandas:

Pandas is a popular Python package for data science, and with good reason: it offers powerful, expressive and flexible data structures that make data manipulation and analysis easy, among many other things. The Data Frame is one of these structures.

Pandas is a high-level data manipulation tool developed by Wes McKinney. It is built on the Numpy package and its key data structure is called the Data Frame. Data Frames allow you to store and manipulate tabular data in rows of observations and columns of variables.

Pandas is built on top of the NumPy package, meaning a lot of the structure of NumPy is used or replicated in Pandas. Data in pandas is often used to feed statistical analysis in SciPy, plotting functions from Matplotlib, and machine learning algorithms in Scikit-learn.

\section{Tools Used:}

Visual Studio Code :

VScode is a streamlined code editor with support for development operations like debugging, task running, and version control. It aims to provide just the tools a developer needs for a 
quick code-build-debug cycle and leaves more complex workflows to fuller featured IDEs, such as Visual Studio IDE.

UML Diagrammer:

UML, which represents Unified Modeling Language, is an approach to visually address the engineering, plan, and execution of complex programming frameworks. At the point when you're composing code, there are a huge number of lines in an application, and it's hard to monitor the connections and progressions inside a product framework. UML diagrams partition that product framework into segments and subcomponents.

Jupyter Notebooks:

Jupyter Notebooks offer a good environment for using pandas to do data exploration and modeling, but pandas can also be used in text editors just as easily.

Jupyter Notebooks give us the ability to execute code in a particular cell as opposed to running the entire file. This saves a lot of time when working with large datasets and complex transformations. Notebooks also provide an easy way to visualize pandas' DataFrames and plots. As a matter of fact, this article was created entirely in a Jupyter Notebook.

\section{ALGORITHM FOR PREVENTING SQL INJECTION}

\section{Naïve Bayes Algorithm}

Naïve Bayes algorithm is a supervised learning algorithm, which is based on Bayes theorem and used for solving classification problems. It is mainly used in text classification that includes a high-dimensional training dataset. It is a probabilistic classifier, which means it predicts on the basis of the probability of an object

Naïve: It is called Naïve because it assumes that the occurrence of a certain feature is independent of the occurrence of other features. Such as if the fruit is identified on the bases of color, shape, and taste, then red, spherical, and sweet fruit is recognized as an apple. Hence each feature individually contributes to identify that it is an apple without depending on each other. 
Bayes: It is called Bayes because it depends on the principle of Bayes' Theorem.

Bayes' theorem is also known as Bayes' Rule or Bayes' law, which is used to determine the probability of a hypothesis with prior knowledge. It depends on the conditional probability.

The formula for Bayes' theorem is given as:

$\mathrm{P}(\mathrm{A} \mid \mathrm{B})=\frac{P(B \mid A) P(A)}{P(B)}$

Where,

$\mathrm{P}(\mathrm{A} \mid \mathrm{B})$ is Posterior probability: Probability of hypothesis A on the observed event B.

$\mathrm{P}(\mathrm{B} \mid \mathrm{A})$ is Likelihood probability: Probability of the evidence given that the probability of a hypothesis is true.

$\mathrm{P}(\mathrm{A})$ is Prior Probability: Probability of hypothesis before observing the evidence.

$\mathrm{P}(\mathrm{B})$ is Marginal Probability: Probability of Evidence.

There are different types of Naive Bayes Model, which are given below:

\section{GaussianNB}

A Gaussian Naive Bayes algorithm is a special type of NB algorithm. It's specifically used when the features have continuous values. It's also assumed that all the features are following a gaussian distribution i.e., normal distribution.

In this project we use Jupyter Notebook and the code is established in Python. The classifier is very fast, even it is no big data set, and the accuracy is perfect. Of course with other permutations and other ratio between training and testing set there will be other results.

\section{Steps to implement:}

1. Data Pre-processing step

2. Fitting Naive Bayes to the Training set

3. Predicting the test result 
4. Test accuracy of the result(Creation of Confusion matrix)

5. Visualizing the test set result.

1) Data Pre-processing step:

In this step, we will pre-process/prepare the data so that we can use it efficiently in our code. It is similar as we did in data-pre-processing. The dataset is loaded into the program and the loaded dataset is divided into training and test set, and then we have scaled the feature variable.

2) Fitting Naive Bayes to the Training Set:

After the pre-processing step, now we will fit the Naive Bayes model to the Training set. Here GaussianNB classifier is used to fit the training dataset. We can also use other classifiers as per our requirement.

3) Prediction of the test set result:

Here we can predict the test set result. For this, we will create a new predictor variable pred, and will use the predict function to make the predictions.

\section{4) Creating Confusion Matrix:}

In this step we will check the accuracy of the Naive Bayes classifier using the Confusion matrix.

A confusion matrix is a table that is often used to describe the performance of a classification model (or "classifier") on a set of test data for which the true values are known. The confusion matrix itself is relatively simple to understand, but the related terminology can be confusing.

5) Visualizing the training set and test set result:

In this step we visualize the training set result using Naïve Bayes Classifier. In the output the Naïve Bayes classifier has segregated the data points with the fine boundary. There will be some wrong predictions which is calculated in Confusion matrix. But still it is pretty good classifier. 


\section{K-Nearest Neighbor (KNN) Algorithm}

$\mathrm{K}-\mathrm{NN}$ algorithm assumes the similarity between the new case/data and available cases and put the new case into the category that is most similar to the available categories. KNN can be used for both classification and regression predictive problems. However, it is more widely used in classification problems in the industry. The " $\mathrm{K}$ " is $\mathrm{KNN}$ algorithm is the nearest neighbor we wish to take the vote from.

To evaluate any technique we generally look at 3 important aspects:

1. Ease to interpret output

2. Calculation time

3. Predictive Power

\section{Breaking it Down - Pseudo Code of KNN}

We can implement a KNN model by following the below steps:

1. Load the data

2. Initialise the value of $\mathrm{k}$

3. For getting the predicted class, iterate from 1 to total number of training data points

1. Calculate the distance between test data and each row of training data. Here we will use Euclidean distance as our distance metric since it's the most popular method. The other metrics that can be used are Chebyshev, cosine, etc.

2. Sort the calculated distances in ascending order based on distance values

3. Get top k rows from the sorted array

4. Get the most frequent class of these rows

5. Return the predicted class

\subsection{TEST DRIVEN DEVELOPMENT}

Test Driven Development, or TDD, is a code design technique where the programmer writes a test before any production code, and then writes the code that will make that test pass. The idea is that with a tiny bit of assurance from that initial test, the programmer can feel free to refactor and refactor some more to get the cleanest code they know how to write. The idea is simple, but like most simple things, the execution is hard. TDD requires a completely different 
mindset from what most people are used to and the tenacity to deal with a learning curve that may slow you down at first.

\section{Functional Testing types include:}

- Unit Testing

- Integration Testing

- System Testing

- Sanity Testing

- Smoke Testing

- Interface Testing

- Regression Testing

Non-functional Testing types include:

- Performance Testing

- Load Testing

- Stress Testing

- Volume Testing

- Security Testing

- Compatibility Testing

- Install Testing

- Recovery Testing

- Reliability Testing

- Usability Testing

- Compliance Testing

- Localization Testing

\section{UNIT TESTING}

UNIT TESTING is a level of software testing where individual units/ components of a software are tested. The purpose is to validate that each unit of the software performs as designed. A unit is the smallest testable part of any software. It usually has one or a few inputs and usually a single output.

In procedural programming, a unit may be an individual program, function, procedure, etc. In object-oriented programming, the smallest unit is a method, which may belong to a base/ super 
class, abstract class or derived/ child class. (Some treat a module of an application as a unit. This is to be discouraged as there will probably be many individual units within that module.) Unit testing frameworks, drivers, stubs, and mock/ fake objects are used to assist in unit testing.

\section{BLACK BOX TESTING}

During functional testing, testers verify the app features against the user specifications. This is completely different from testing done by developers which is unit testing. It checks whether the code works as expected. Because unit testing focuses on the internal structure of the code, it is called the white box testing. On the other hand, functional testing checks app's functionalities without looking at the internal structure of the code, hence it is called black box testing. Despite how flawless the various individual code components may be, it is essential to check that the app is functioning as expected, when all components are combined. Here you can find a detailed comparison between functional testing vs unit testing.

\section{INTEGRATION TESTING}

INTEGRATION TESTING is a level of software testing where individual units are combined and tested as a group. The purpose of this level of testing is to expose faults in the interaction between integrated units. Test drivers and test stubs are used to assist in Integration Testing.

Integration testing: Testing performed to expose defects in the interfaces and in the interactions between integrated components or systems. See also component integration testing, system integration testing.

Component integration testing: Testing performed to expose defects in the interfaces and interaction between integrated components. System integration testing: Testing the integration of systems and packages; testing interfaces to external organizations (e.g. Electronic Data Interchange, Internet). 


\section{SYSTEM TESTING}

SYSTEM TESTING is a level of software testing where a complete and integrated software is tested. The purpose of this test is to evaluate the system's compliance with the specified requirements. System Testing means testing the system as a whole. All the modules/components are integrated in order to verify if the system works as expected or not.

System Testing is done after Integration Testing. This plays an important role in delivering a highquality product.

\section{SANITY TESTING}

Sanity Testing is done when as a QA we do not have sufficient time to run all the test cases, be it Functional Testing, UI, OS or Browser Testing. Sanity testing is a subset of regression testing. After receiving the software build, sanity testing is performed to ensure that the code changes introduced are working as expected. This testing is a checkpoint to determine if testing for the build can proceed or not.

The main purpose of this testing is to determine that the changes or the proposed functionality are working as expected. If the sanity test fails, the build is rejected by the testing team to save time and money. It is performed only after the build has cleared the smoke test and been accepted by the Quality Assurance team for further testing.

Smoke Testing is done to make sure if the build we received from the development team is testable or not. It is also called as "Day 0" check. It is done at the "build level". It helps not to waste the testing time to simply testing the whole application when the key features don't work or the key bugs have not been fixed yet. Here our focus will be on primary and core application work flow.

To conduct smoke testing, we don't write test cases. We just pick the necessary test cases from already written test cases.

\section{REGRESSION TESTING}

Regression Testing is a type of testing that is done to verify that a code change in the software does not impact the existing functionality of the product. This is to make sure the product works 
fine with new functionality, bug fixes or any change in the existing feature. Previously executed test cases are re-executed in order to verify the impact of change.

Regression Testing is a Software Testing type in which test cases are re-executed in order to check whether the previous functionality of the application is working fine and the new changes have not introduced any new bugs.

\section{LOAD TESTING}

Load Testing is type of performance testing to check system with constantly increasing the load on the system until the time load is reaches to its threshold value. Here Increasing load means increasing number of concurrent users, transactions \& check the behavior of application under test. It is normally carried out underneath controlled environment in order to distinguish between two different systems. It is also called as "Endurance testing" and "Volume testing".

The main purpose of load testing is to monitor the response time and staying power of application when system is performing well under heavy load. Load testing comes under the Non Functional Testing \& it is designed to test the non-functional requirements of a software application. 


\section{CHAPTER 5 \\ FUTURE SCOPE AND CONCLUSION}

\section{FUTURE SCOPE}

As per the user Requirement our whole project is designed. We can add an additional constraint to our project. We will also try to make the modification, update, delete, any other facility in our project. This can be used in property management as well as for other commercial purpose.

Some of them are:-

- Business relationship with comprehensive online services.

- Affiliate Marketing Systems, Web site Design, and Development and Search Engine optimization.

- Integration with other standard Application Software Products \&Booking Engines / Platforms, Fare \& Content Management Systems

\section{CONCLUSION}

I was able to understand the importance of Planning and Designing as a part of software development. The system provide major advantages such as speed and accuracy of operation, Time Efficiency, Cost Efficiency, Automatic data validation, Data security and reliability, Easy performance check ,Dynamic and User Friendly. Generates real-time, comprehensive reports and ensures access to complete and critical information, instantly.

The proposed system detects SQLIA by analyzing log files. The main idea of the model is to leverage a grammatical recognizer and behavioral miner to extract two kinds of features automatically. The evaluation of the model is done using five different machine learning algorithms based on these two training sets. 


\section{APPENDIX A}

\section{SAMPLE SOURCE CODE}

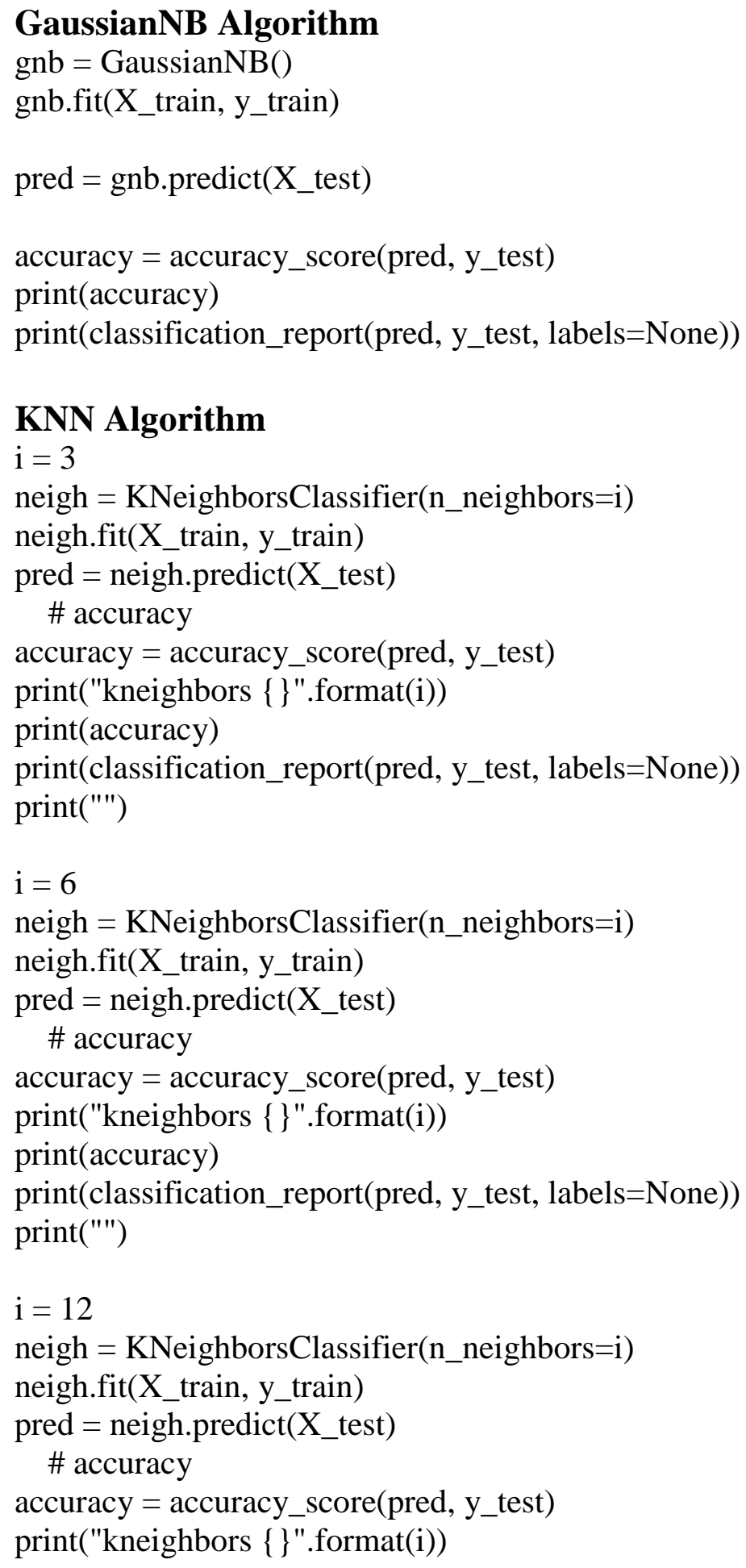


print(accuracy)

print(classification_report(pred, y_test, labels=None))

print("')

$\mathrm{i}=15$

neigh $=$ KNeighborsClassifier(n_neighbors=i)

neigh.fit(X_train, y_train)

pred $=$ neigh.predict $\left(X \_\right.$test $)$

\# accuracy

accuracy = accuracy_score(pred, y_test)

print("kneighbors \{\} ".format(i))

print(accuracy)

print(classification_report(pred, y_test, labels=None))

print("")

\section{HTML}

〈div class="preloader - it" >

$\langle$ div class="la-anim-1" $\rangle\langle$ div $\rangle$

$\langle/$ div $\rangle$

$\langle$ !-- /Preloader -- >

〈div class="wrapper theme-1-active pimary-color-green">

<div class="bg-image"

style="background-image: url('logo.jpg');

height: 100vh">

$\langle/$ div $>$

$<$ ! -- Top Menu Items -->

〈nav class="navbar navbar-inverse navbar-fixed-top">

〈div class="mobile-only-brand pull-left">

〈div class="nav-header pull-left">

〈div class="logo-wrap">

〈a href="index.html">

<span class="brand-text" $>$ Admin $</$ span $>$

$\langle/ a\rangle$

$\langle/$ div $\rangle$

$\langle/$ div $\rangle$

$<$ id="toggle_nav_btn" class="toggle-left-nav-btn inline-

block ml-20 pull-left" href="javascript:void(0);" $><$ class="zmdi zmdi-

menu" $>\langle/ i\rangle\langle/ a\rangle$

<a id="toggle_mobile_search" data-toggle="collapse" datatarget="\#search_form" class="mobile-only-

view" href="javascript:void(0);" $\rangle\langle$ i class="zmdi zmdi-search" $\rangle\langle/ i\rangle\langle/ a\rangle$ 
<a id="toggle_mobile_nav" class="mobile-only-

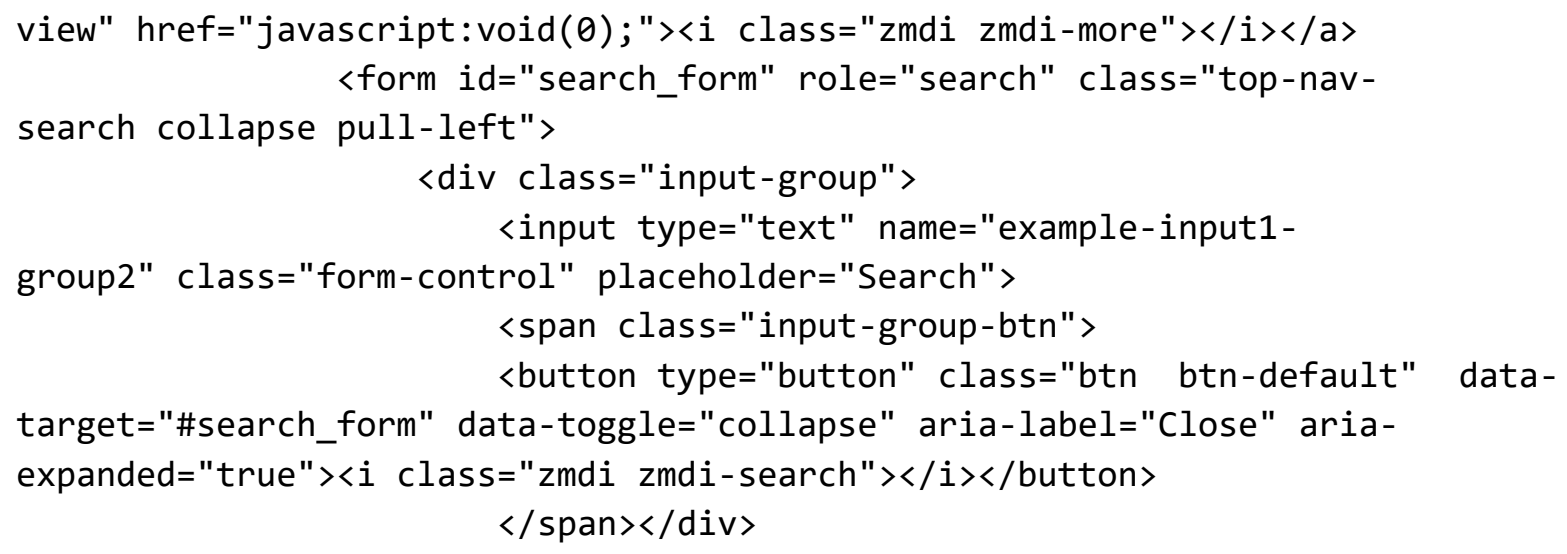

\section{CSS}

input \{

outline: none;

border: none;

\}

textarea \{

outline: none;

border: none;

\}

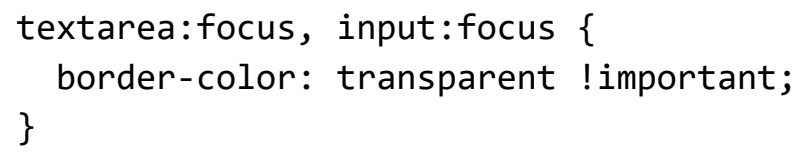




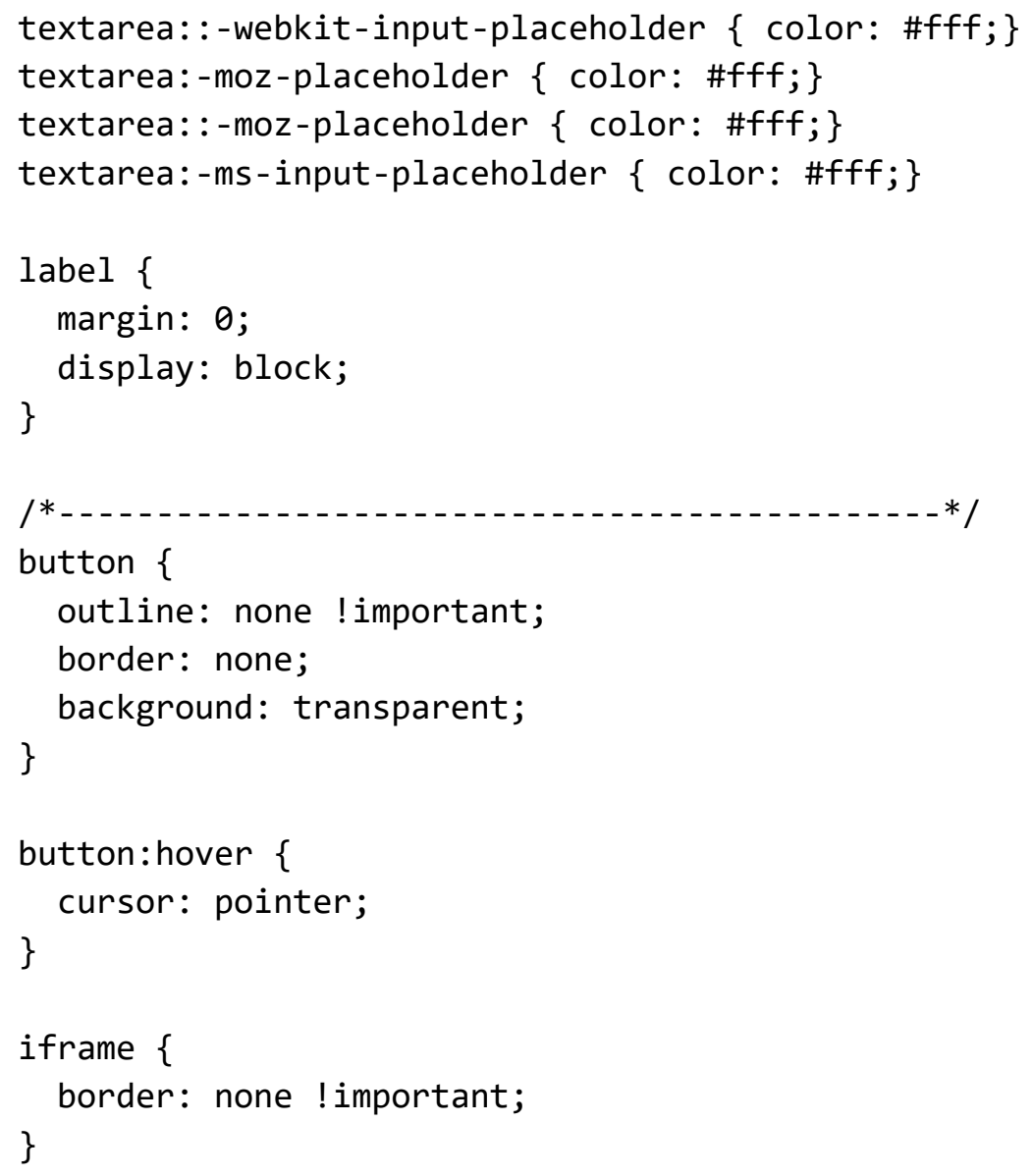

\section{JavaScript}

(function $(\$)\{$

"use strict";

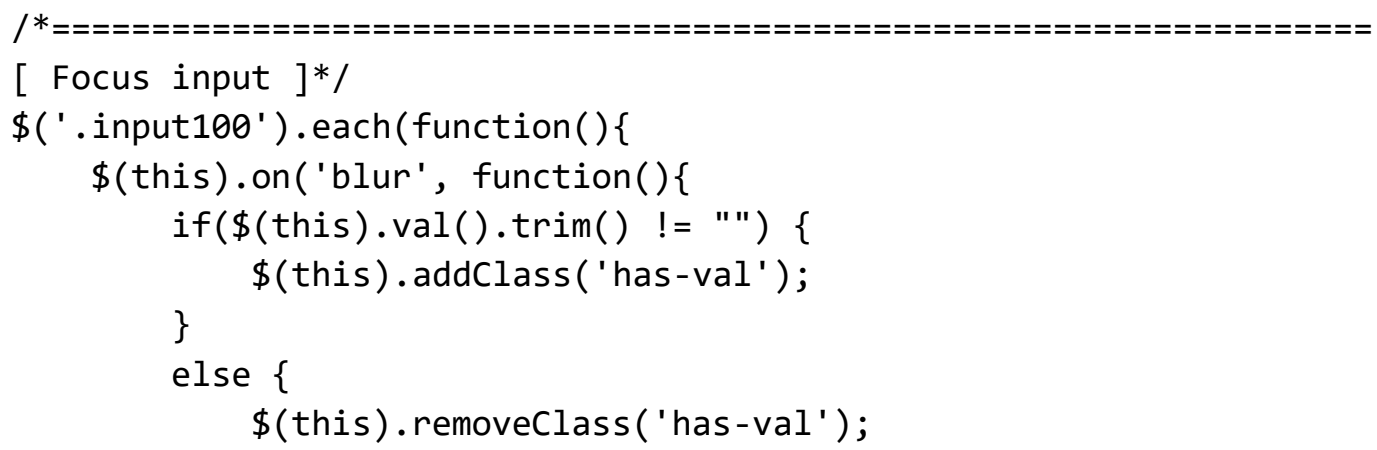




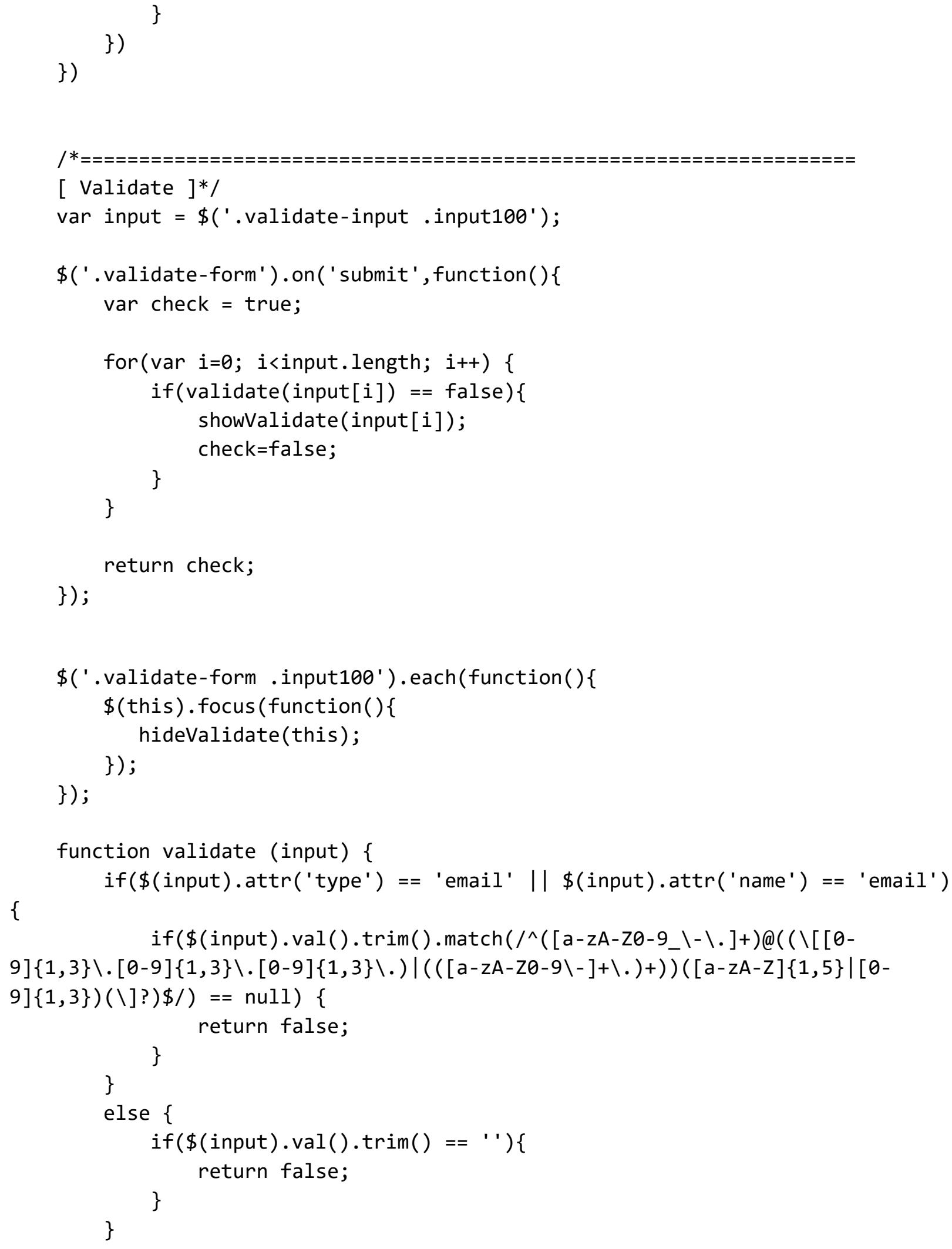


\}

function showValidate(input) \{

var thisAlert $=\$($ input $) \cdot \operatorname{parent}()$;

$\$$ (thisAlert) . addClass( 'alert-validate' );

\}

function hideValidate(input) \{

var thisAlert $=\$$ (input) . parent () ;

$\$$ (thisAlert). removeclass( 'alert-validate' );

\}

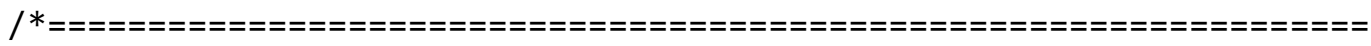

[ Show pass ]*/

var showPass $=0$;

$\$($ '.btn-show-pass').on('click', function()\{

if ( showPass $==\theta)\{$

$\$$ (this).next('input'). attr('type', 'text');

$\$$ (this). addClass ('active');

\}

showPass = 1 ;

else \{

$\$$ (this).next('input').attr ('type' , 'password' ');

$\$$ (this). removeclass('active');

showPass $=0$;

\}

\});

\})(jQuery); 


\section{APPENDIX B}

\section{SCREENSHOTS}
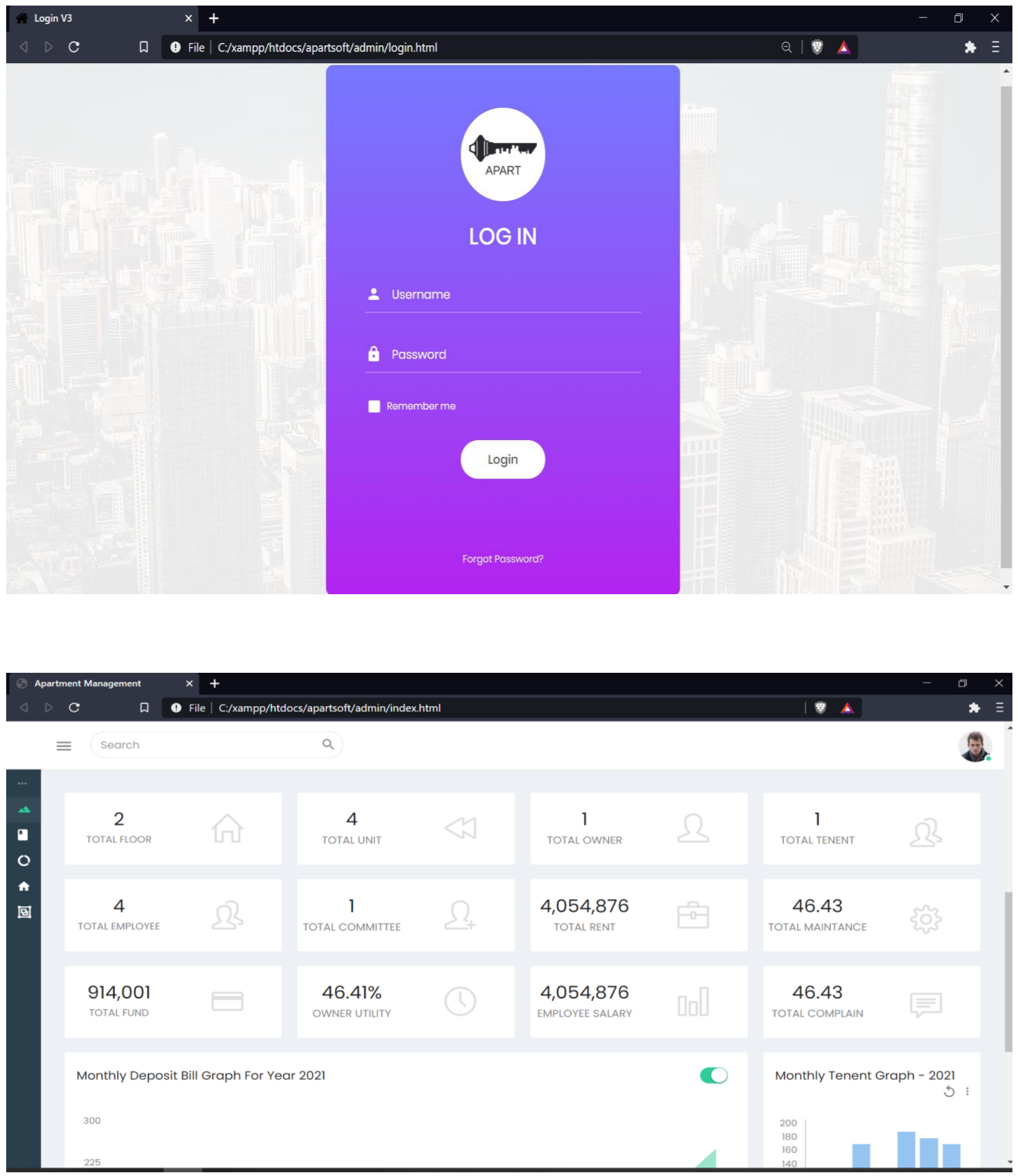

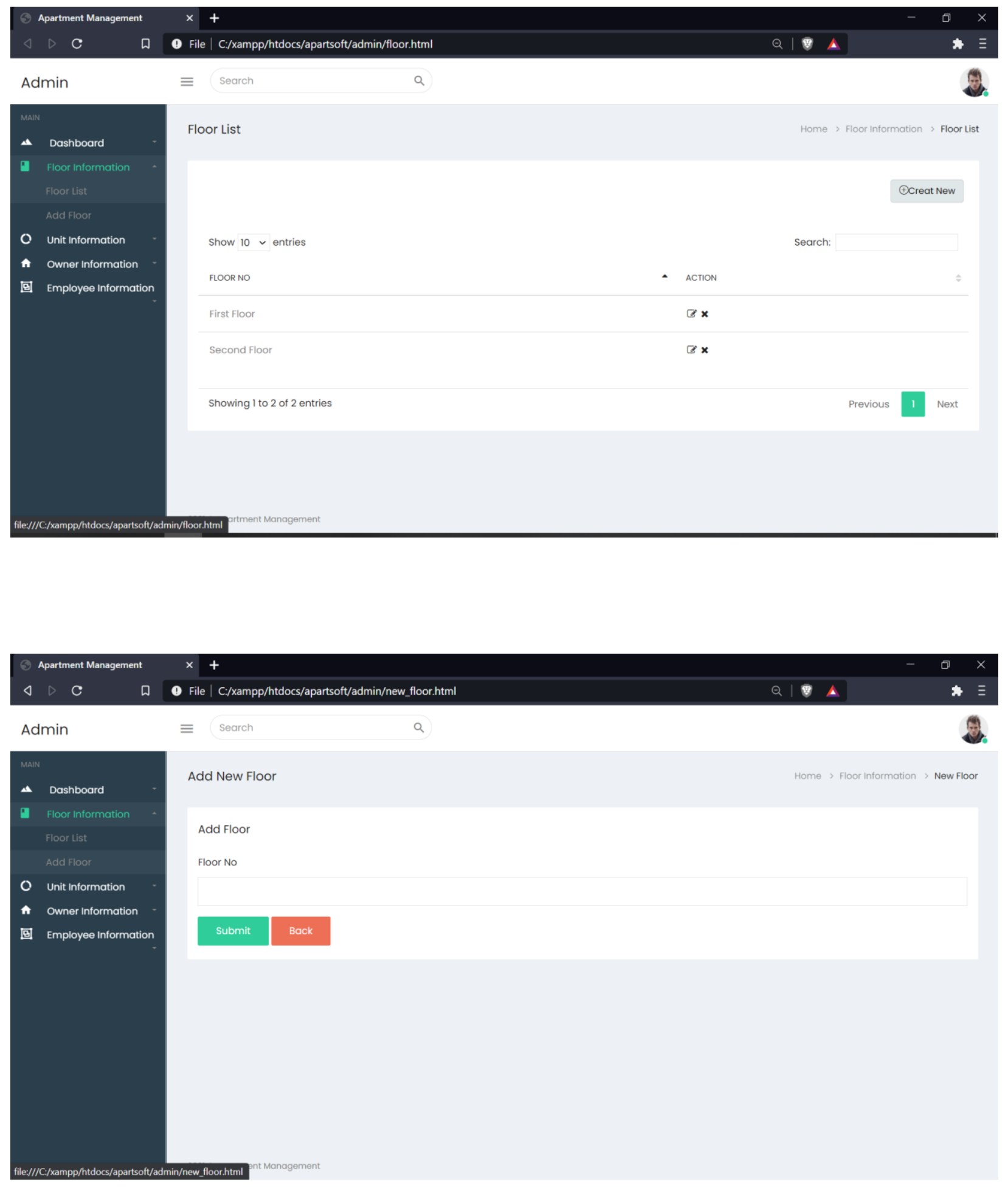

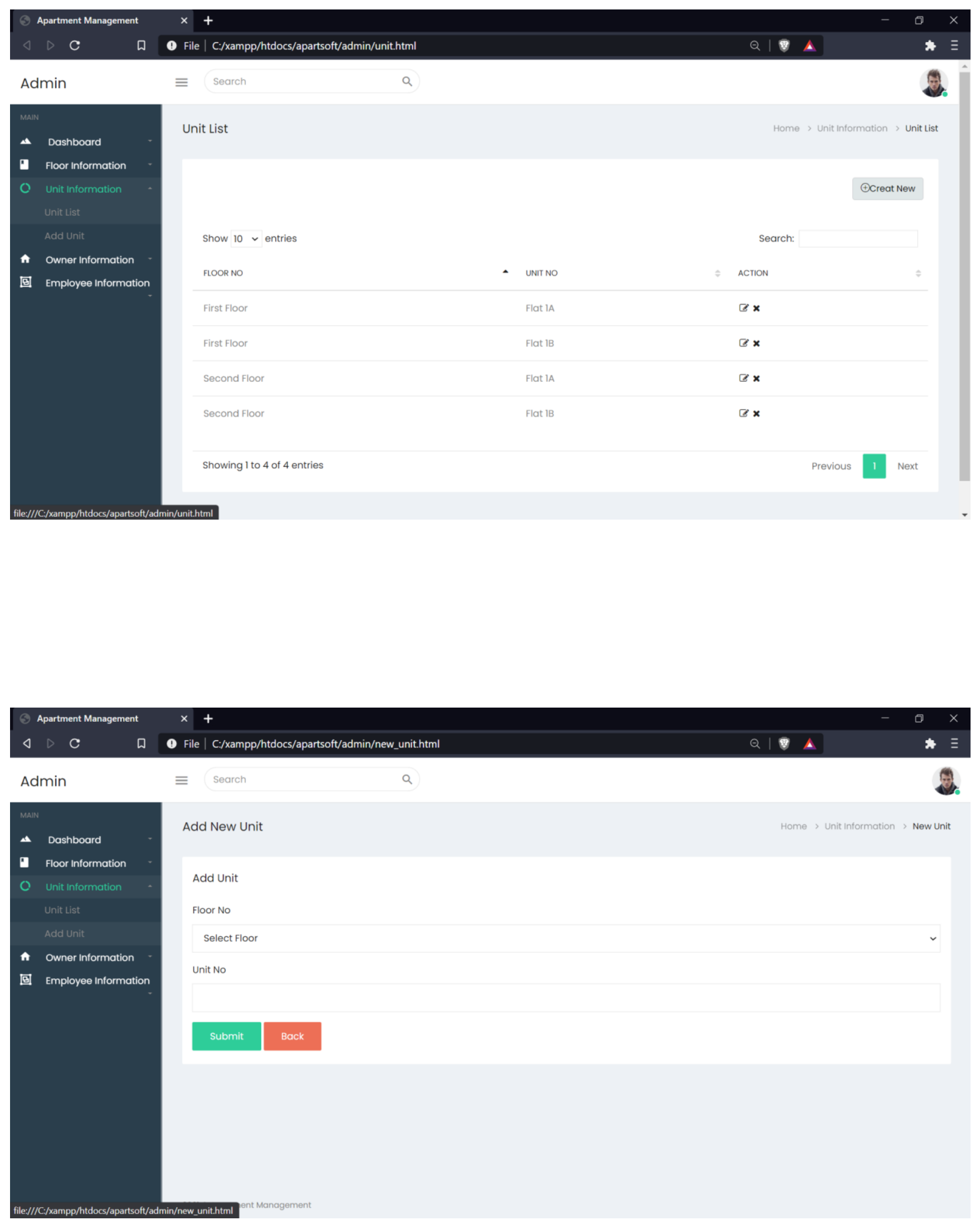

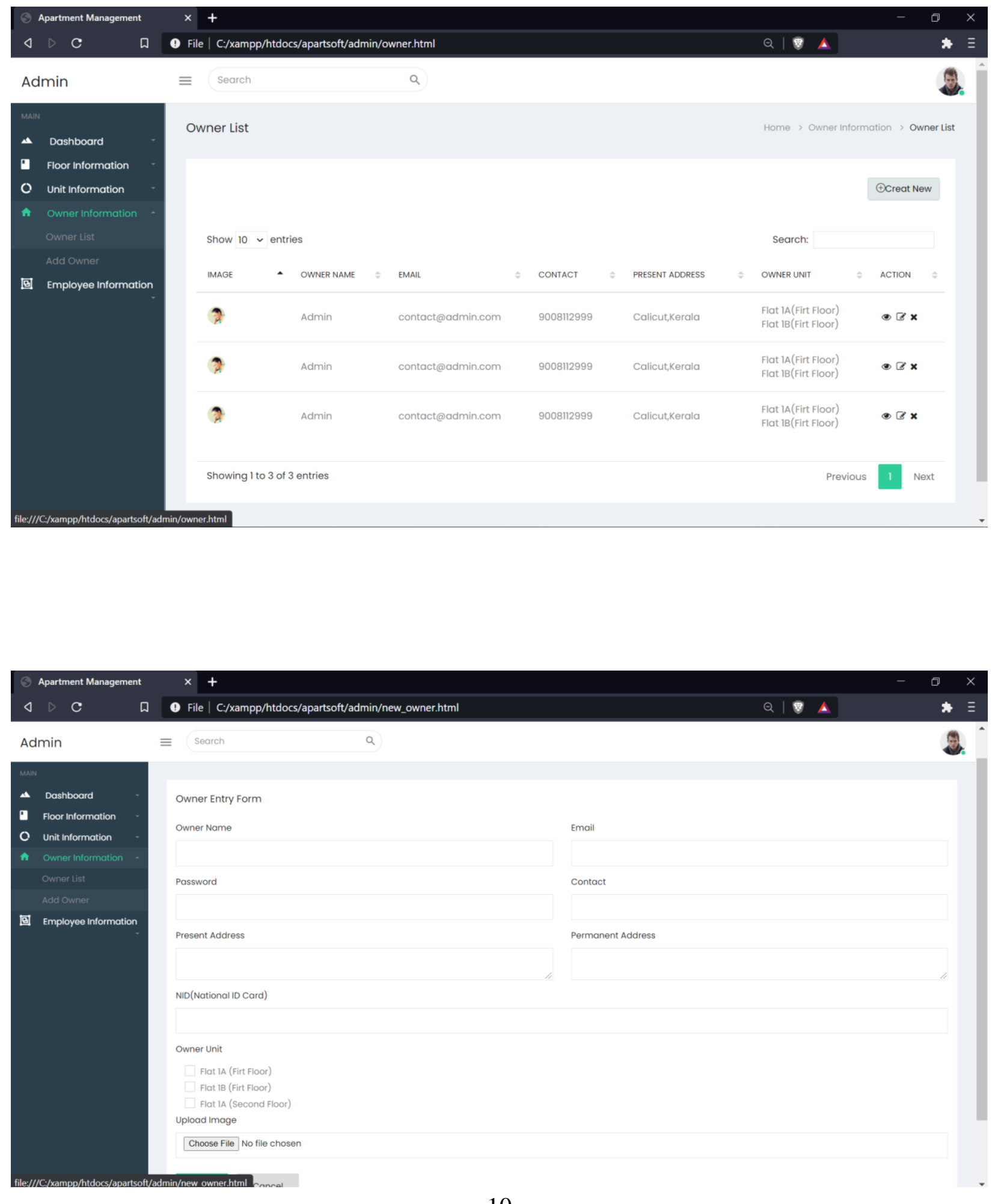


\section{GaussianNB Algorithm}

1.0

$\begin{array}{rrrrr} & \text { precision } & \text { recall } & \text { f1-score } & \text { support } \\ 0 & 1.00 & 1.00 & 1.00 & 34 \\ 1 & 1.00 & 1.00 & 1.00 & 46\end{array}$

accuracy

macro avg

weighted avg

1.00

1.00

80

1.00

1.00

80

1.00

1.00

1.00

80

\section{KNN Algorithm}

kneighbors 3

0.9625

$\begin{array}{rrrrr} & \text { precision } & \text { recall } & \text { f1-score } & \text { support } \\ 0 & 1.00 & 0.92 & 0.96 & 37 \\ 1 & 0.93 & 1.00 & 0.97 & 43\end{array}$

accuracy

macro avg

weighted avg

$\begin{array}{llll} & & 0.96 & 80 \\ 0.97 & 0.96 & 0.96 & 80 \\ 0.96 & 0.96 & 0.96 & 80\end{array}$


kneighbors 6 0.9625

$\begin{array}{rrrrr} & \text { precision } & \text { recall } & \text { f1-score } & \text { support } \\ 0 & 1.00 & 0.92 & 0.96 & 37 \\ 1 & 0.93 & 1.00 & 0.97 & 43 \\ & & & & \\ \text { cy } & & & 0.96 & 80 \\ \text { vag } & 0.97 & 0.96 & 0.96 & 80 \\ \text { avg } & 0.96 & 0.96 & 0.96 & 80\end{array}$

kneighbors 12

0.9625 precision recall f1-score support

$\begin{array}{lllll}0 & 1.00 & 0.92 & 0.96 & 37 \\ 1 & 0.93 & 1.00 & 0.97 & 43\end{array}$

$\begin{array}{rllll}\text { accuracy } & & & 0.96 & 80 \\ \text { macro avg } & 0.97 & 0.96 & 0.96 & 80 \\ \text { weighted avg } & 0.96 & 0.96 & 0.96 & 80\end{array}$


kneighbors 15

0.9625

$\begin{array}{rrrrr} & \text { precision } & \text { recall } & \text { f1-score } & \text { support } \\ 0 & 1.00 & 0.92 & 0.96 & 37 \\ 1 & 0.93 & 1.00 & 0.97 & 43 \\ & & & & \\ \text { cy } & & & 0.96 & 80 \\ \text { vg } & 0.97 & 0.96 & 0.96 & 80 \\ \text { vg } & 0.96 & 0.96 & 0.96 & 80\end{array}$




\section{REFERENCES}

1. D. Mitropoulos, P. Louridas, M. Polychronakis, and A. D. Keromytis, “'Defending against Web application attacks: Approaches, challenges and implications," IEEE Trans. Depend. Sec. Comput., vol. 16, no. 2, pp. 188203, Mar./Apr. 2017.

2. E. Pollack, 'Protecting against SQL injection: Applications, performance, and security in microsoft SQL server," in Proc. Dyn. SQL, 2019, pp. 3160.

3. Maraj, E. Rogova, G. Jakupi, and X. Grajcevci, “'Testing techniques and analysis of SQL injection attacks," in Proc. Int. Conf. Knowl. Eng. Appl. (ICKEA), 2017, pp. 111.

4. Sarhan, S. A. Farhan, and F. M. Al-Harby, ' Understanding and discovering SQL injection vulnerabilities," in Proc. Int. Conf. Appl. Hum. Factors Ergonom., 2017, pp. 10631075.

5. D. Das, U. Sharma, and D. K. Bhattacharyya, “Defeating SQL injection attack in authentication security: An experimental study," Int. J. Inf. Secur., vol. 18, no. 1, pp. 122, 2017.

6. H.-C. Huang, Z.-K. Zhang, H.-W. Cheng, and S. W. Shieh, “Web applica- tion security: Threats, countermeasures, and pitfalls," Computer, vol. 50, no. 6, pp. 8185, 2017.

7. P. R. McWhirter, K. Kifayat, Q. Shi, and B. Askwith, ''SQL injection attack classification through the feature extraction of SQL query strings using a gap- weighted string subsequence kernel," J. Inf. Secur. Appl., vol. 40, pp. 199216, Jun. 2018.

8. B J. Santhosh Kumar and P. P. Anaswara, "Vulnerability detection and prevention of SQL injection," Int. J. Eng. Technol., vol. 7, no. 2.31, pp. 1618, 2018.

9. "Smart Phone," [Online]. Available: http://www.idc.com/prodserv/smartphone-osmarketshare.jsp. [Accessed 2015].

10. "Wikipedia.org. Android. [Online].," [Online]. Available:

11. http://en.wikipedia.org/wiki/Android_(operating_system). [Accessed 10 May 2015].

12. "Android Studio," [Online]. Available:

13. http://developer.android.com/tools/studio/index.html. [Accessed ND].

14. "Android Developers[Online]," [Online]. Available:

15. http://developer.android.com/training/basics/firstapp/index.html. [Accessed ND].

16. "Parse Android Docs. [Online].," [Online]. Available: 
17. https://www.parse.com/docs/android_guide. [Accessed ND].

18. "Squared Away Chicago web based app.," [Online]. Available: http://www.tenantsrights.

19. org/press-release-squared-away-chicago-renters-app-now-on-line/. [Accessed ND]. 